\title{
Seasonal radiative modeling of Titan's stratospheric temperatures at low latitudes
}

\author{
Bruno Bézard $^{\mathrm{a}^{*}}$, Sandrine Vinatier ${ }^{\mathrm{a}}$, Richard K. Achterberg ${ }^{\mathrm{b}}$
}

${ }^{a}$ : LESIA, Observatoire de Paris, PSL Research University, CNRS, Sorbonne Universités, UPMC Univ. Paris 6, Université Paris-Diderot, Sorbonne Paris Cité, 5 place Jules Janssen, 92195 Meudon, France

b: University of Maryland, Department of Astronomy, College Park, MD 20742, United States

* Corresponding author.

E-mail address: bruno.bezard@obspm.fr

Contact details: $\quad$ Bruno Bézard

LESIA, Bât. 18

Observatoire de Paris, section de Meudon

92195 Meudon cedex

France

Phone: (33) 145077717

Fax: (33) 145072806

Published in Icarus, Volume 302, 1 March 2018, Pages 437-450

https://doi.org/10.1016/j.icarus.2017.11.034 
3 We have developed a seasonal radiative-dynamical model of Titan's stratosphere to

4 investigate the temporal variation of temperatures in the $0.2-4$ mbar range observed by the

5 Cassini/CIRS spectrometer. The model incorporates gas and aerosol vertical profiles derived

6 from Cassini/CIRS and Huygens/DISR data to calculate the radiative heating and cooling rate

7 profiles as a function of time and latitude. At $20^{\circ} \mathrm{S}$ in 2007 , the heating rate is larger than the

8 cooling rate at all altitudes, and more specifically by $20-35 \%$ in the $0.1-5$ mbar range. A new

9 calculation of the radiative relaxation time as a function of pressure level is presented, leading

10 to time constants significantly lower than previous estimates. At $6^{\circ} \mathrm{N}$ around spring equinox,

11 the radiative equilibrium profile is warmer than the observed one at all levels. Adding

12 adiabatic cooling in the energy equation, with a vertical upward velocity profile

13 approximately constant in pressure coordinates below the $0.02-\mathrm{mbar}$ level (corresponding to

$14 \quad 0.03-0.05 \mathrm{~cm} \mathrm{~s}^{-1}$ at $1 \mathrm{mbar}$ ), allows us to reproduce the observed profile quite well. The

15 velocity profile above the $\sim 0.5$-mbar level is however affected by uncertainties in the haze

16 density profile. The model shows that the change in insolation due to Saturn's orbital

17 eccentricity is large enough to explain the observed 4-K decrease in equatorial temperatures

18 around 1 mbar between 2009 and 2016 . At $30^{\circ} \mathrm{N}$ and $\mathrm{S}$, the radiative model predicts seasonal

19 variations of temperature much larger than observed. A seasonal modulation of adiabatic

20 cooling/heating is needed to reproduce the temperature variations observed from 2005 to 2016

21 between 0.2 and 4 mbar. At 1 mbar, the derived vertical velocities vary in the range -0.05

22 (winter solstice) to 0.16 (summer solstice) $\mathrm{cm} \mathrm{s}^{-1}$ at $30^{\circ} \mathrm{S},-0.01$ (winter solstice) to 0.14

23 (summer solstice) $\mathrm{cm} \mathrm{s}^{-1}$ at $30^{\circ} \mathrm{N}$, and $0.03-0.07 \mathrm{~cm} \mathrm{~s}^{-1}$ at the equator.

25 Key words: Titan, atmosphere; Atmospheres, structure; Atmospheres, dynamics 


\section{Introduction}

28 Due to Saturn's obliquity of $26.7^{\circ}$, Titan experiences large seasonal variations of insolation. The 0.056 eccentricity of Saturn's orbit adds a significant modulation to this insolation. Above the 10-mbar level, Titan's radiative time constant is less than a Titan year (29.5 Earth years) (Strobel et al. 2009, Flasar et al. 2014) so that significant seasonal variations of temperature are expected in the mid-stratosphere and mesosphere.

Infrared observations by the IRIS instrument aboard the Voyager 1 spacecraft in November 1980 pointed out a north-to-south asymmetry of temperatures in the $0.4-1$ mbar region, with temperatures at $55^{\circ} \mathrm{S}$ being higher than at $55^{\circ} \mathrm{N}$ by 4 and $8 \mathrm{~K}$ at 1 and 0.4 mbar respectively (Flasar et al. 1990). These observations occurred shortly after northern spring equinox, at a heliocentric longitude $L_{\mathrm{s}} \approx 9^{\circ}$. Flasar and Conrath (1990) proposed that the asymmetry was due to a phase lag in the response of the atmosphere to the seasonally-varying insolation due to dynamical inertia. On the other hand, Bézard et al. (1995) suggested that the asymmetry results from the larger concentrations of infrared radiators (photochemical gases and aerosols) present at high northern latitudes.

The Cassini Composite Infrared Spectrometer (CIRS) aboard Cassini allowed us to monitor the thermal structure of Titan's stratosphere from July 2004 to September 2017, which corresponds to $L_{\mathrm{s}} \approx 293^{\circ}$. Combining limb- and nadir-viewing observations between 2004 and 2006, Achterberg et al. (2008a) retrieved the temperature field over the pressure range $5 \times 10^{-3}$ 5 mbar from about $75^{\circ} \mathrm{S}$ to $75^{\circ} \mathrm{N}$. The corresponding season was around northern mid-winter $\left(L_{\mathrm{s}}=293-323^{\circ}\right)$. Compared with Voyager 1 observations, the north-to-south asymmetry was stronger and temperatures at $55^{\circ} \mathrm{S}$ were higher than at $55^{\circ} \mathrm{N}$ by about 18 and $11 \mathrm{~K}$ at 1 and 0.4 mbar respectively. Compared to southern latitudes, high northern latitudes were then 
experiencing reduced solar heating and enhanced abundances of photochemical gases and aerosols, both of which likely contribute to the lower temperatures. Besides this asymmetry, mid-stratosphere temperatures on Titan were reaching their maximum at latitudes $0-30^{\circ} \mathrm{S}$. On the other hand, the stratopause was found higher and warmer beyond $50^{\circ} \mathrm{N}$ than anywhere else on the satellite, which very likely results from adiabatic heating from downwelling air at winter polar latitudes. Achterberg et al. (2011) extended the analysis of Achterberg et al. (2008a) using Cassini/CIRS data up to December 2009, i.e. shortly after northern spring equinox $\left(L_{\mathrm{s}} \approx 4^{\circ}\right)$. Between 2004 and 2009, a large decrease of temperatures in the stratopause region (above the 0.1 -mbar level) was found beyond $30^{\circ} \mathrm{N}$. Elsewhere in the stratosphere and lower mesosphere, the temperature variations did not exceed $5 \mathrm{~K}$.

The temporal and latitudinal variations of temperature observed in the stratosphere and lower mesosphere result from combined variations of the insolation, modulating the solar heating rate, of the atmospheric composition, which governs the radiative cooling and solar heating rates, and of dynamical motions, which provide adiabatic heating and cooling. To try to assess the relative importance of these actors, it is first necessary to constrain as precisely as possible the radiative forcing terms, which requires a good knowledge of the distribution of the radiatively-active gases and aerosols. Such information is available from Cassini/CIRS, which measures in nadir- and limb-viewing geometry the thermal emission spectrum of Titan from 10 to $1495 \mathrm{~cm}^{-1}$. This allows the retrieval of the gas concentration and aerosol extinction profiles that contribute to the radiative cooling between approximately 130 and $450 \mathrm{~km}$ (50.005 mbar) (e.g. Vinatier et al. 2010a, 2010b, 2015). The Descent Imager/Spectral Radiometer (DISR) aboard the Huygens probe measured the optical properties and vertical distribution of haze particles between 0 and $\approx 150 \mathrm{~km}$ (Tomasko et al. 2008c, Doose et al. 2016) on 14 January 2005 near $10^{\circ} \mathrm{S}$. Used with a correct representation of the methane 
opacity, these results allow us to compute the solar heating rate profile as a function of zenith angle. Combining Huygens/DISR and Cassini/CIRS data, Tomasko et al. (2008b) were able to investigate the heat balance at the location and time of the Huygens descent. They inferred that the day-averaged solar heating rate profile exceeded the cooling rate profile by a maximum of $0.5 \mathrm{~K} /$ Titan day $(0.03 \mathrm{~K} /$ Earth day $)$ near $120 \mathrm{~km}$ altitude $(5.5 \mathrm{mbar})$ and concluded that the general circulation must redistribute this heat to higher latitudes.

In Titan's stratosphere, a meridional circulation, similar to Hadley cells on Earth, is driven by the latitude-dependent solar heating (see a review of Titan's general circulation in Lebonnois et al. 2014). General Circulation Models (GCMs) have been developed to investigate Titan's dynamics, in particular the superrotation characterized by prograde zonal winds up to $\sim 200 \mathrm{~m}$ $\mathrm{s}^{-1}$ in the winter stratosphere (see Newman et al. 2011, Lebonnois et al. 2012 and Lora et al. 2015 for recent three-dimensional GCMs). These models show a pole-to-pole circulation, particularly in the stratosphere, with rising motion in the summer hemisphere and subsidence in the winter hemisphere except around equinox, when a more symmetric equator-to-pole circulation takes place throughout the atmosphere. They generally succeed in reproducing at least qualitatively the dominant features of Titan's atmospheric structure, such as the zonal wind pattern and temperature field, but suffer from approximations in the treatment of the radiative transfer and/or various other simplifications. The strong subsidence at high winter latitudes predicted by the GCMs is confirmed by the high temperatures and the large enrichment in minor photochemical species observed in the upper stratosphere and mesosphere (Achterberg et al. 2011; Teanby et al. 2007, 2009; Vinatier et al. 2007, 2010a). The temperature anomalies observed in winter around the north pole have been used to estimate downward vertical velocities of $\sim 10 \mathrm{~cm} \mathrm{~s}^{-1}$ around $0.01 \mathrm{mbar}$ in 2005-2007 (Achterberg et al. 2011). Changes in the vertical abundance profiles of minor species 
observed near the south pole in autumn were also used to derive the following vertical

103 velocities: from 0.1 to $0.4 \mathrm{~cm} \mathrm{~s}^{-1}$ near 0.003 mbar in 2010-2011 and 2011-2012 (Teanby et al.

104 2012, Vinatier et al. 2015), $0.25 \mathrm{~cm} \mathrm{~s}^{-1}$ near $0.01 \mathrm{mbar}$ in 2011-2012, and $0.4 \mathrm{~cm} \mathrm{~s}^{-1}$ near 0.02

105 mbar in 2015 (Vinatier et al. 2017a).

106

107 The goal of this paper is to investigate the heat balance of Titan's stratosphere using a

108 seasonal radiative model based on measurements by Cassini/CIRS of the distributions of the

109 radiative agents and state-of-the-art representation of gas and aerosol spectral properties. We

110 also take into account constraints from Huygens/DISR measurements. We then calculate the

111 season-dependent radiative solution for the temperature profile and compare it with the

112 observed variations of temperature at different levels and latitudes to derive constraints on the

113 dynamical heating/cooling. Here, we restrain our analysis to mid-latitudes $\left(30^{\circ} \mathrm{S}-30^{\circ} \mathrm{N}\right)$ where

114 gas and aerosol do not exhibit large seasonal variations. Section 2 describes the temperature

115 data, retrieved from Cassini/CIRS measurements, with which we are comparing our model.

116 Section 3 presents our seasonal radiative model and the gas and aerosol distributions used in

117 the radiative transfer code to calculate heating and cooling rates. Radiative model results are

118 presented in Section 4 and compared with observations to constrain the missing adiabatic

119 heating and cooling terms. Also shown is a calculation of the radiative time constant as a

120 function of pressure level. We discuss these results in Section 5 and present our conclusions

121 in Section 6.

\section{2. Observations}

124 Retrievals of Titan's temperature field are routinely achieved using nadir and limb

125 observations of the $v_{4}$ band of methane through Focal Plane FP4 of Cassini/CIRS. This focal

126 plane covers the interval $1050-1495 \mathrm{~cm}^{-1}$ at a spectral resolution adjustable from 15.5 to 0.5 
$127 \mathrm{~cm}^{-1}$ (apodized). It consists of a 10-pixel linear array, with a 0.27-mrad field of view (FOV)

128 per pixel (Flasar et al. 2004). Temperature maps were retrieved by Achterberg et al. (2008a)

129 for mid-winter conditions (2004-2006) combining nadir-viewing (2.8- $\mathrm{cm}^{-1}$ resolution) and

130 limb-viewing $\left(15.5-\mathrm{cm}^{-1}\right.$ resolution) sequences. The nadir data cover latitudes from $90^{\circ} \mathrm{S}$ to

$13160^{\circ} \mathrm{N}$ and yield information in a pressure range of about 5-0.2 mbar while the limb data cover

132 latitudes from $75^{\circ} \mathrm{S}$ to $85^{\circ} \mathrm{N}$ and yield information in a pressure range $\approx 1-0.005$ mbar.

133 Achterberg et al. (2011) extended the analysis to cover 5.5 years of Cassini/CIRS

134 observations from July 2004 to December 2009, just after northern spring equinox. Here we

135 use a further extended data set encompassing observations up to June 2016, i.e. Titan flybys

$136 \mathrm{~T} 0$ to $\mathrm{T} 118$ (Achterberg et al. in preparation). For each flyby, zonal-mean temperatures were

137 obtained by zonally averaging temperatures retrieved from individual nadir-viewing spectra

138 (2.8- $\mathrm{cm}^{-1}$ resolution) using $5^{\circ}$ latitude bins with $2.5^{\circ}$ spacing and interpolating the retrieved

139 temperatures onto a uniform latitude grid for each flyby. Averaging was done in a reference

140 frame that removes the $4^{\circ}$ offset of the stratospheric symmetry axis from the surface pole

141 (Achterberg et al. 2008b). In our analysis, we used temperatures retrieved at 0.2, 0.5, 1, 2 and

1424 mbar, which cover the range of maximum temperature information. Note that these

143 temperatures actually represent a vertical average over 1 to 1.5 scale heights due to the width

144 of the contribution functions in the methane band and the filtering applied in the retrieval

145 process. We restrained our analysis to equatorial and mid-latitudes and selected data at $\theta=0^{\circ}$,

$14630^{\circ} \mathrm{N}$ and $30^{\circ} \mathrm{S}$. For a given latitude $\theta$ and a given flyby, we averaged the three temperatures

147 retrieved by Achterberg et al. (in preparation) at latitudes $\theta-2.5^{\circ}, \theta$ and $\theta+2.5^{\circ}$. Figure 1

148 shows the retrieved 1-mbar temperatures as a function of time for these three latitudes. The

149 error bars correspond to the standard deviation (SD) of temperatures in each $5^{\circ}$ bin divided by

150 square of root of the number of data points. Seasonal variations are clearly visible in this data

151 set. At the equator, the 1-mbar $(\sim 185 \mathrm{~km})$ temperature increases slightly from 2005 to 2011 - 
1522012 (by less than $1 \mathrm{~K}$ ), and decreases more noticeably after 2012 (by $\sim 4 \mathrm{~K}$ between 2012

153 and 2016). At $30^{\circ} \mathrm{N}$, the temperature increases by $\sim 4 \mathrm{~K}$ from 2005 to 2012 and decreases by

154 about the same amount from 2012 to 2016 , while at $30^{\circ} \mathrm{S}$ the temperature regularly decreases

155 by $\sim 8 \mathrm{~K}$ from 2005 to 2016.

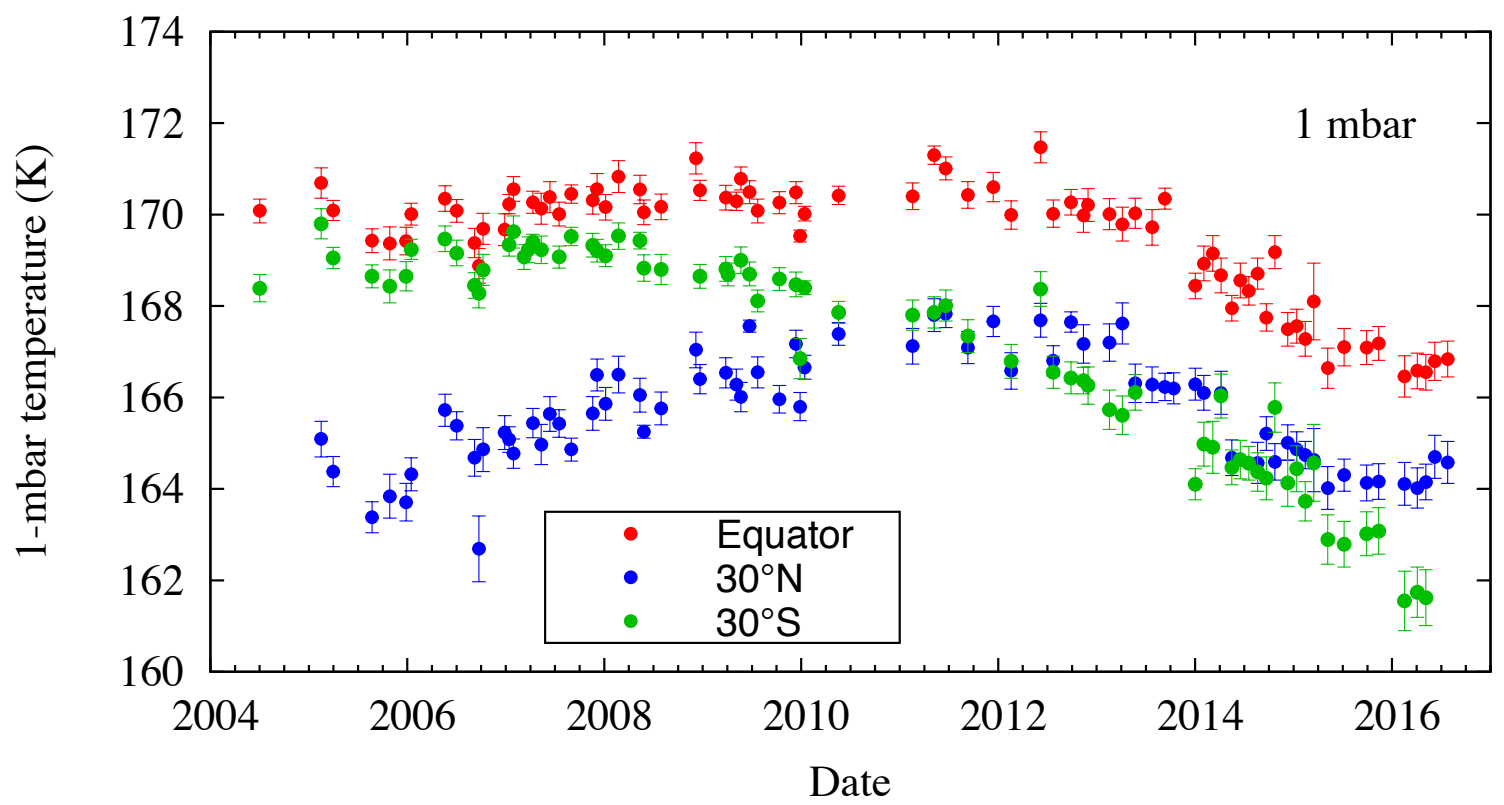

156

Figure 1: Time variation of 1-mbar temperatures retrieved from Cassini/CIRS measurements at three latitudes, $0^{\circ}, 30^{\circ} \mathrm{N}$ and $30^{\circ} \mathrm{S}$.

158 Vinatier et al. (2015) produced maps of temperature and composition from selected sequences

159 of CIRS limb spectra recorded between October 2006 and May 2013 at a resolution of either

$160 \quad 0.5$ or $15.5 \mathrm{~cm}^{-1}$. Reliable information extends from 5 to $0.001 \mathrm{mbar}$, a region below which

161 the temperature profile smoothly joins the Huygens/HASI profile measured in situ at $10^{\circ} \mathrm{S}$ in

162 January 2005 (Fulchignoni et al. 2005). We used Vinatier et al.'s results around $6^{\circ} \mathrm{N}$, a

163 latitude least sensitive to seasonal effects, as a reference profile to test our model and

164 investigate the heat balance at this latitude. To smooth out small local temperature variations,

165 we averaged four temperature profiles recorded at $4-5^{\circ} \mathrm{N}$, on January $2007\left(\mathrm{Ls}=327^{\circ}\right)$,

166 December $2009\left(\mathrm{Ls}=4^{\circ}\right)$, June $2010\left(\mathrm{Ls}=11^{\circ}\right)$ and May $2011\left(\mathrm{Ls}=21^{\circ}\right)$, thus corresponding 167 to northern mid-winter to mid-spring conditions. This profile is shown in Fig. 7. The 1-SD 
168 formal error bar due to noise propagation is about $\pm 0.2 \mathrm{~K}$ in the range $0.1-1$ mbar increasing

169 to $\pm 0.3 \mathrm{~K}$ at $0.01 \mathrm{mbar}$ and $\pm 0.4 \mathrm{~K}$ at 5 mbar. The standard deviation of our set of four

170 temperature profiles is larger, 0.5 to $1.5 \mathrm{~K}$ from 0.2 to 2 mbar, $2 \mathrm{~K}$ at 0.1 and 5 mbar and $5 \mathrm{~K}$

171 at 0.01 mbar.

172

173 3. Seasonal radiative model

174 We developed a one-dimensional seasonal radiative-dynamical model to investigate the

175 observed temperature variations in Titan's stratosphere. We solve for the energy equation:

$176 \quad \frac{\partial T(z)}{\partial t}=h(z)-c(z)-w(z)\left(\frac{g(z)}{C_{p}}+\frac{\partial T(z)}{\partial z}\right)$.

$177 h(z)$ is the solar heating rate equal to $-\frac{g}{C_{p}} \frac{d F_{*}(p)}{d p}$, where $F *$ is the downward solar flux, $c(\mathrm{z})$ is

178 the radiative cooling rate equal to $-\frac{g}{C_{p}} \frac{d F_{I R}(p)}{d p}$ with $F_{I R}$ being the upward thermal emission

179 flux, $w$ the upward vertical velocity, $C_{p}$ the specific heat, and $g$ the acceleration of gravity.

180 The term $w(z) \frac{g(z)}{C_{p}}$ represents the adiabatic cooling rate and $w(z) \frac{\partial T(z)}{\partial z}$ the cooling rate due to

181 vertical advection. The solar flux is calculated for diurnally averaged (i.e. zonally-averaged)

182 insolation. We neglect the meridional advection of heat. As discussed by Achterberg et al.

183 (2011) and Teanby et al. (2017), this term is expected to be $\leq 0.2$ time the vertical advection

184 term, based on the mass continuity equation and the observed horizontal temperature

185 gradients, all the more at low latitudes where these temperature gradients are very small.

186

3.1 Solar flux

188 Our atmospheric grid consists of 41 pressure levels uniformly distributed in log-scale from

1891.466 bar (surface pressure) to $0.133 \mu$ bar (around $650 \mathrm{~km}$ ). The solar flux is calculated as a

190 function of zenith angle $\theta_{s}$ and altitude from 2610 to $40000 \mathrm{~cm}^{-1}(3.8-0.25 \mu \mathrm{m})$ using a plane

191 parallel radiative transfer code that incorporates the DISORT algorithm (Stamnes et al. 1988) 
with 8 streams to solve for scattering. The solar irradiance spectrum at 1 AU is the 2000 ASTM Standard Extraterrestrial Spectrum Reference E-490-00 (available at http://rredc.nrel.gov/solar/spectra/am0/). We consider opacity from methane and aerosols. Methane absorption is modeled from $2610 \mathrm{~cm}^{-1}(3.8 \mu \mathrm{m})$ to $25000 \mathrm{~cm}^{-1}(0.400 \mu \mathrm{m})$ using the correlated- $k$ distribution method. From 2690 to $11850 \mathrm{~cm}^{-1}$, absorption coefficients are calculated with a line-by-line radiative transfer model and molecular line parameters (positions, intensities, energy levels) from the TheoReTS database, which includes new accurate theoretical linelists of ${ }^{12} \mathrm{CH}_{4},{ }^{13} \mathrm{CH}_{4}$ and ${ }^{12} \mathrm{CH}_{3} \mathrm{D}$ (Rey et al. 2017). The assumed $\mathrm{N}_{2}$ broadened halfwidths and far wing lineshape are described in Rey et al. The $\mathrm{CH}_{3} \mathrm{D} / \mathrm{CH}_{4}$ ratio corresponds to $\mathrm{D} / \mathrm{H}=1.32 \times 10^{-4}$ (Bézard et al. 2007). For each $40-\mathrm{cm}^{-1}$ interval, a set of $16 k$ coefficients, 8 for the interval [0:0.95] of the normalized frequency $g$ and 8 for the interval [0.95:1.00], is calculated on a set of pressures and temperatures. Beyond $11850 \mathrm{~cm}^{-1}$, we used the coefficients of the Voigt-Goody band model calculated by Karkoschka and Tomasko (2010, Table 4) based on methane transmission measurements from laboratory, Huygens and HST data. We then essentially proceeded as in Irwin et al. (1996) and generated, for each pressure and temperature of our set, 24 transmission $\left(T_{r}\right)$ spectra with column densities $(a)$ equally spaced in log space between a minimum value such as $T_{r} \approx 0.997$ and a maximum value such as $T_{r} \leq 0.01$. This function $T_{r}(a)$ was then fitted with an exponential sum characterized by 10-point Gaussian Legendre quadrature abscissae and weights, using a Levenberg-Marquardt non-linear least squares algorithm (Press et al. 1997). The first guess of the $10 k_{i}$ absorption coefficients was derived from the $k$ distribution of a Malkmus-Lorentz band model (Eq. 12 of Irwin et al. 1996) having the $S$ and $B$ parameters given in Table 4 of Karkoschka and Tomasko (2010). The function $T_{r}(a)$ was actually fitted with the 10 parameters $k_{1}$ and $\left(k_{i}-k_{i-1}\right)$, for $\mathrm{i}=2,10$, discarding the iterations leading to negative values of any of them, to ensure that they increase monotonically. We kept the original sampling of 
217 Karkoschka and Tomasko (2010): $5 \mathrm{~cm}^{-1}$ in the interval $11850-19300 \mathrm{~cm}^{-1}$ and $25 \mathrm{~cm}^{-1}$ in the 218 interval $19300-25000 \mathrm{~cm}^{-1}$.

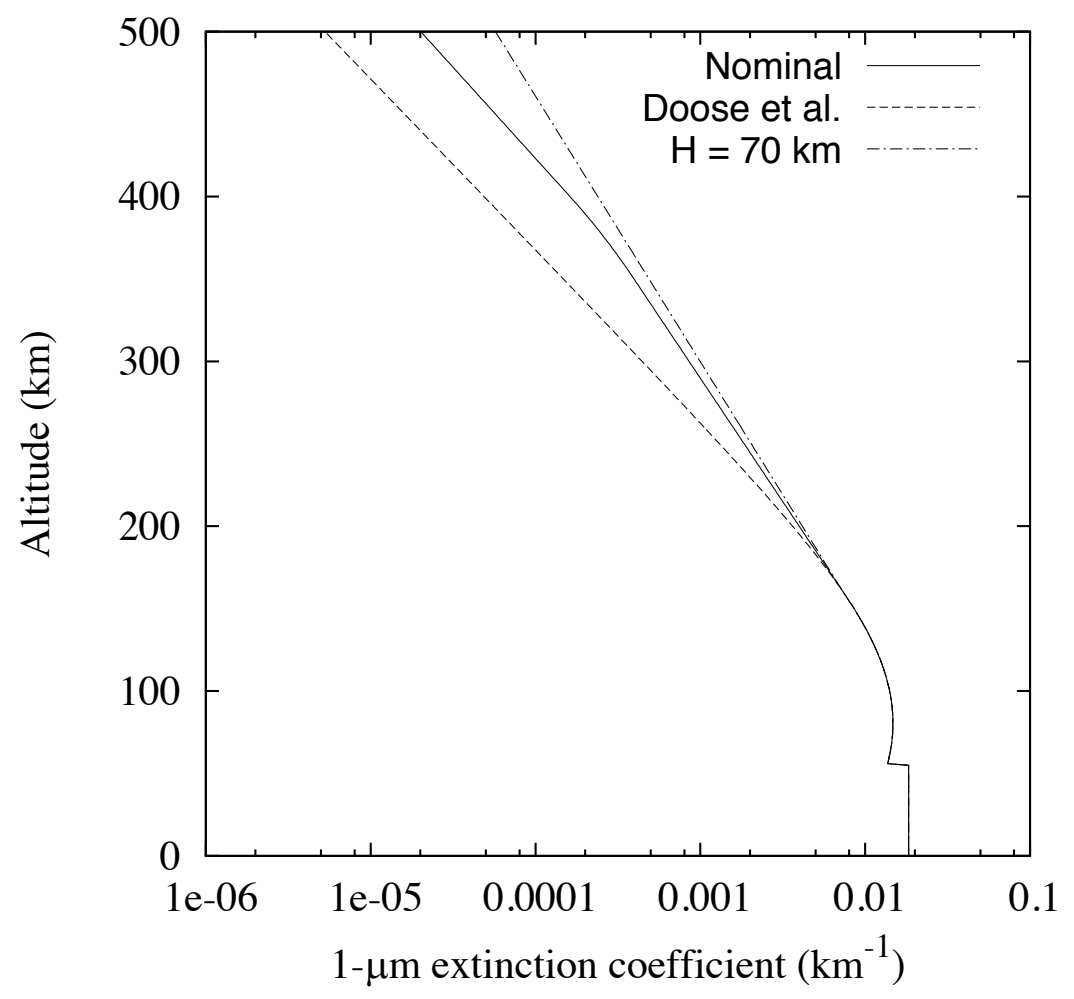

Figure 2: Haze extinction coefficient at $1 \mu \mathrm{m}$ for our nominal model (solid line), Doose et al.'s (2016) model (dashed line) and one with a constant scale height of $70 \mathrm{~km}$ above 160 $\mathrm{km}$ (dash-dot line). In the thermal infrared range, at $1090 \mathrm{~cm}^{-1}$, these extinction profiles are scaled by factors of $0.0103,0.0113$ and 0.0099 respectively.

220 The methane mole fraction profile used in the radiative transfer is that derived by Niemann et

221 al. (2010) from the analysis of Huygens/GCMS in situ measurements, with a uniform mole

222 fraction of $1.48 \times 10^{-2}$ above $45 \mathrm{~km}$. The temperature profile $T_{0}(p)$ is that retrieved by Vinatier

223 et al. (2010a) from Cassini/CIRS limb and nadir spectra recorded near $20^{\circ} \mathrm{S}$ in March 2007,

224 not far from the Huygens descent latitude.

226 The properties of the aerosol particles (single scattering albedo, phase function) were taken

227 from the recent reanalysis of Huygens/DISR observations by Doose et al. (2016, Table 2) 
with information on the phase function from Tomasko et al. (2008c). Longward of $950 \mathrm{~nm}$, we used the single scattering albedo derived by Hirtzig et al. (2013) from modeling of Cassini/VIMS data. We used the wavelength dependence of the extinction given in Table 2 of Doose et al. (2016). The vertical variation of the extinction at the Huygens site is constrained in detail between 0 and $144 \mathrm{~km}$ from the DISR measurements but only in average above this altitude. Doose et al. then added the additional constraint of the optical limb altitude as observed by Karkoschka and Lorenz (1997) to produce an analytical vertical profile of extinction characterized, in the stratosphere, by an optical depth scale height decreasing from large values below $80 \mathrm{~km}$ to a value of $65 \mathrm{~km}$ at $120 \mathrm{~km}$ and an asymptotic value of $45 \mathrm{~km}$ at very high altitudes (Fig. 2). Here we considered additional constraints from Cassini/CIRS measurements of the aerosol continuum emission in limb-viewing and nadir geometry between 600 and $1500 \mathrm{~cm}^{-1}$. These measurements provide a vertical profile of the extinction at thermal wavelengths with a good precision between approximately 140 and $400 \mathrm{~km}$ (3-0.01 mbar) (Vinatier et al. 2010b, 2015). We used here the vertical dependence of the extinction determined from limb observations in January 2007 around $5^{\circ} \mathrm{N}$ (Vinatier et al. 2010a, b) and adapted by Vinatier et al. (2015) (dashed line in their Fig. 15). Their extinction profile has a scale height $(H)$ of about $\sim 65 \mathrm{~km}$ up to $350 \mathrm{~km}$ decreasing to $\sim 48 \mathrm{~km}$ above $400 \mathrm{~km}$. Our nominal profile for the haze extinction is then the Doose et al. profile up to $160 \mathrm{~km}$, that we extend above with $H=65 \mathrm{~km}$ from 160 to $350 \mathrm{~km}$, linearly decreasing to $48 \mathrm{~km}$ at $400 \mathrm{~km}$ (Fig. 2). The extinction profiles derived from CIRS measurements at equatorial and midlatitudes show however a significant variability with latitude and year above $250 \mathrm{~km}$ (Vinatier et al. 2015, 2016). Although part of it may be an artifact due to uncertainties in the continuum calibration, especially at high altitudes ( $\geq 400 \mathrm{~km})$, most of it is probably real, including the presence of the variable detached haze layer, as discussed in Vinatier et al. (2015). An average of the profiles observed in $2010-2012$ between $20^{\circ} \mathrm{N}$ and $30^{\circ} \mathrm{S}$ (Fig. 15 of Vinatier et 
al. 2015) shows a vertical variation close to the Doose et al. profile up to $300 \mathrm{~km}$ and

254 intermediate between our nominal profile and Doose et al. up to $400 \mathrm{~km}$. On the other hand, 255 observations at $26^{\circ} \mathrm{S}$ in January 2016 exhibit an approximately constant scale height $H$ as 256 large as $70 \mathrm{~km}$ between 200 and $500 \mathrm{~km}$ (Vinatier et al. 2016). We consider that the full 257 Doose et al. profile and one having $H=70 \mathrm{~km}$ above $160 \mathrm{~km}$ represent a reasonable envelope 258 of the actual extinction profiles at low latitudes (Fig. 2).

We assumed a Lambertian surface with the reflectivity inferred by Jacquemart et al. (2008) between 900 and $1600 \mathrm{~nm}$ from DISR/DLIS spectra taken at an altitude of $25 \mathrm{~m}$ and after landing of the Huygens probe. The surface reflectivity down to $400 \mathrm{~nm}$ was obtained from Eq. 5 of Karkoschka et al. (2012), giving the relative spectral variation of I/F derived from DISR/DLVS data after landing, and scaled with the Jacquemart et al. value at $900 \mathrm{~nm}$.

Between 250 and $400 \mathrm{~nm}$, we assumed a linear variation of $4 \times 10^{-4} \mathrm{~nm}^{-1}$ and, beyond 1600 nm, we used the Hirtzig et al. (2013) albedos derived from the methane windows in

267 Cassini/VIMS spectra near the Huygens landing site. We account neither for the strong opposition effect seen in the phase function of Titan's surface (Karkoschka et al. 2012) nor for the fact that the surface at Huygens' landing site is darker than average at low latitudes.

270 However, the influence of the surface reflectivity on the heating rate at stratospheric levels 271 should be relatively low.

\subsection{Thermal emission}

274 Without scattering, the thermal flux at pressure level $p$ is equal to:

$F_{I R}(p)=2 \pi \int_{0}^{\infty} d \sigma\left[\int_{\tau_{\sigma}}^{\infty} B_{\sigma}\left(T\left(\tau_{\sigma}^{\prime}\right)\right) E_{2}\left(\tau_{\sigma}^{\prime}-\tau_{\sigma}\right) d \tau_{\sigma}^{\prime}-\int_{0}^{\tau_{\sigma}} B_{\sigma}\left(T\left(\tau_{\sigma}^{\prime}\right)\right) E_{2}\left(\tau_{\sigma}-\tau_{\sigma}^{\prime}\right) d \tau_{\sigma}^{\prime}\right]$,

276 where $\tau_{\sigma}$ is the optical depth at wavenumber $\sigma$ and pressure level $p, B_{\sigma}\left(\tau_{\sigma}{ }^{\prime}\right)$ is the Planck

277 function at the temperature $T$ of the level of optical depth $\tau_{\sigma}{ }^{\prime}$ and wavenumber $\sigma$, and $E_{2}$ is 
278 the second-order exponential integral $\left(E_{2}(x)=\int_{1}^{\infty} \frac{e^{-x t}}{t^{2}} d t\right)$. The two terms in Eq. 2 represent 279 the fluxes from respectively the upwelling and downwelling radiation at pressure level $p$. We 280 calculate this integral from 20 to $1560 \mathrm{~cm}^{-1}$, and divide it into $n_{k}=74$ intervals of width $\delta \sigma=$ $28120 \mathrm{~cm}^{-1}$. Thermal emission from outside this spectral interval can be neglected in the energy 282 budget. Our grid has $n_{p}=51$ pressure levels uniformly distributed in log-scale from $p_{1}$ $283=1.466$ bar (surface pressure) to $p_{n_{p}}=0.1466 \mu$ bar (around $650 \mathrm{~km}$ ). By linearizing the 284 Planck function as a function of optical depth within each atmospheric layer of our grid $[j$, $285 j+1$, and assuming that the Planck function is constant over each spectral interval $k$ of width $286 \delta \sigma$, i.e.:

$B_{\sigma_{k}}\left(T\left(\tau_{\sigma}^{\prime}\right)\right)=B_{\sigma_{k}}\left(T\left(p_{j}\right)\right) \frac{\tau_{\sigma}\left(p_{j+1}\right)-\tau_{\sigma}^{\prime}}{\tau_{\sigma}\left(p_{j+1}\right)-\tau_{\sigma}\left(p_{j}\right)}+B_{\sigma_{k}}\left(T\left(p_{j+1}\right)\right) \frac{\tau_{\sigma}^{\prime}-\tau_{\sigma}\left(p_{j}\right)}{\tau_{\sigma}\left(p_{j+1}\right)-\tau_{\sigma}\left(p_{j}\right)}$,

288 Eq. 2 at a given pressure level $p_{i}$ can be written out as summations over indices $k$ and $j$ as:

$F_{I R}\left(p_{i}\right)=\pi \delta \sigma \sum_{k=1}^{n_{k}}\left[\frac{B_{\sigma_{k}}\left(T\left(p_{1}\right)\right)}{\delta \sigma} \int_{\sigma_{k}-\delta \sigma / 2}^{\sigma_{k}+\delta \sigma / 2} 2 E_{2}\left(\tau_{\sigma}\left(p_{1}\right)-\tau_{\sigma}\left(p_{i}\right)\right) d \sigma+\right.$

$\sum_{j=1}^{i-1} \frac{1}{\delta \sigma} \int_{\sigma_{k}-\delta \sigma / 2}^{\sigma_{k}+\delta \sigma / 2} d \sigma \int_{\tau_{\sigma}\left(p_{j+1}\right)}^{\tau_{\sigma}\left(p_{j}\right)} B_{\sigma_{k}}\left(T\left(\tau_{\sigma}^{\prime}\right)\right) 2 E_{2}\left(\tau_{\sigma}^{\prime}-\tau_{\sigma}\left(p_{i}\right)\right) d \tau_{\sigma}^{\prime}-$

$\left.\sum_{j=i}^{n_{p}-1} \frac{1}{\delta \sigma} \int_{\sigma_{k}-\delta \sigma / 2}^{\sigma_{k}+\delta \sigma / 2} d \sigma \int_{\tau_{\sigma}\left(p_{j+1}\right)}^{\tau_{\sigma}\left(p_{j}\right)} B_{\sigma_{k}}\left(T\left(\tau_{\sigma}^{\prime}\right)\right) 2 E_{2}\left(\tau_{\sigma}\left(p_{i}\right)-\tau_{\sigma}^{\prime}\right) d \tau_{\sigma}^{\prime}\right]$

292 where the first term in the summation over $k$ is the contribution from the surface, the second 293 one that from atmospheric layers below level $i$ and the third one that from atmospheric layers 294 above level $i$. Combining Eqs. 3 and 4 allows us to express the flux at pressure level $p_{i}$ as a 295 linear combination of the Planck functions at the $n_{p}$ pressure levels $\left(p_{j}\right)$ and $n_{k}$ wavenumbers $296\left(\sigma_{k}\right)$ :

$F_{I R}\left(p_{i}\right)=\pi \delta \sigma \sum_{k=1}^{n_{k}} \sum_{j=1}^{n_{p}} B_{\sigma_{k}}\left(T\left(p_{j}\right)\right) A_{i, j, k}$

298 where $A_{i, j, k}$ is a dimensionless coupling factor between levels $p_{i}$ and $p_{j}$ for the $k^{\text {th }}$ frequency 299 interval. We calculated this exchange matrix $A$ for the reference temperature profile $T_{0}(p)$ (see 
above) and neglect its dependence on temperature in our seasonal model, given that it is

301 generally much weaker than that of the Planck function.

303 The exchange matrix $A$ was calculated through a line-by-line radiative transfer program that 304 incorporates opacity from the collision-induced absorption (CIA) of $\mathrm{N}_{2}-\mathrm{H}_{2}-\mathrm{CH}_{4}$ pairs, lines 305 from $\mathrm{CH}_{4}, \mathrm{CH}_{3} \mathrm{D}, \mathrm{C}_{2} \mathrm{H}_{6}, \mathrm{C}_{2} \mathrm{H}_{2}, \mathrm{C}_{2} \mathrm{H}_{4}, \mathrm{CH}_{3} \mathrm{C}_{2} \mathrm{H}, \mathrm{C}_{4} \mathrm{H}_{2}, \mathrm{C}_{3} \mathrm{H}_{8}, \mathrm{CO}, \mathrm{CO}_{2}$ and $\mathrm{HCN}$, and aerosols. 306 Spectroscopic line parameters are described in Vinatier et al. (2010a) with, in addition, $\mathrm{C}_{2} \mathrm{H}_{6}$ 307 lines in the 7- $\mu$ m region from HITRAN2012 (Rothman et al. 2013), the $\mathrm{CH}_{3} \mathrm{C}_{2} \mathrm{H}$ bands 308 around 15.5 and $30 \mu \mathrm{m}$ from Geisa2011 (Jacquinet-Husson et al. 2011), and rotational lines 309 from $\mathrm{CH}_{4}, \mathrm{CO}$ and $\mathrm{HCN}$ as described in Lellouch et al. (2014). Line parameters of $\mathrm{C}_{4} \mathrm{H}_{2}$ 310 bands were updated following Geisa2011. References for the CIA coefficients are given in 311 Vinatier et al. (2007), with the $\mathrm{N}_{2}-\mathrm{CH}_{4}$ coefficients multiplied by a factor of 1.5 , following 312 Tomasko et al. (2008b). For the main haze opacity, we utilized the spectral dependence of the 313 extinction cross section derived from Cassini/CIRS measurements by Vinatier et al. (2012) 314 from 600 to $1500 \mathrm{~cm}^{-1}$ and by Anderson and Samuelson (2011) at shorter wavenumbers.

316 We used the vertical profiles of $\mathrm{C}_{2} \mathrm{H}_{6}, \mathrm{C}_{2} \mathrm{H}_{2}, \mathrm{C}_{2} \mathrm{H}_{4}, \mathrm{CH}_{3} \mathrm{C}_{2} \mathrm{H}, \mathrm{C}_{4} \mathrm{H}_{2}, \mathrm{C}_{3} \mathrm{H}_{8}$, and $\mathrm{HCN}$ derived 317 by Vinatier et al. (2010a) from limb measurements near $20^{\circ} \mathrm{S}$ in March $2007^{1}$. As for the 318 calculation of the solar flux, the $\mathrm{CH}_{4}$ profile is that of Niemann et al. (2010) and the $\mathrm{CH}_{3} \mathrm{D}$ 319 profile derives from $\mathrm{D} / \mathrm{H}=1.32 \times 10^{-4}$ (Bézard et al. 2007). The $\mathrm{CO}_{2}$ and $\mathrm{CO}$ mole fractions 320 were held constant at $1.6 \times 10^{-8}$ and $4.7 \times 10^{-5}$ respectively (de Kok et al. 2007).

322 We assumed a uniform composition from $30^{\circ} \mathrm{S}$ to $30^{\circ} \mathrm{N}$ and constant throughout the mission.

323 This is consistent with monitoring studies based on Cassini/CIRS nadir observations by

\footnotetext{
${ }^{1}$ Temperature and abundance profiles available from the Virtual European Solar and Planetary Access (VESPA) http://vespa.obspm.fr/planetary/data/epn/query/all/
} 
324 Teanby et al. (2008) and Coustenis et al. (2013), which all show limited variations of

325 composition at latitudes less than $30^{\circ}$ in the $\sim 2-10$ mbar region. As shown later, $\mathrm{C}_{2} \mathrm{H}_{2}, \mathrm{C}_{2} \mathrm{H}_{6}$

326 and $\mathrm{CH}_{4}$ are the main gaseous cooling agents. Compiling the $\mathrm{C}_{2} \mathrm{H}_{2}$ and $\mathrm{C}_{2} \mathrm{H}_{6}$ profiles retrieved

327 from CIRS limb and nadir observations from 2007 to 2016 by Vinatier et al. (2010a, 2015,

328 2017b), we have calculated the standard deviation (SD) of the mole fractions in these sets at

329 levels between 0.5 and 2 mbar. We found a SD of about $10 \%$ of the mean value for $\mathrm{C}_{2} \mathrm{H}_{6}$,

330 which is about the 1-SD uncertainty of the retrievals, and $15-20 \%$ of the mean value for $\mathrm{C}_{2} \mathrm{H}_{2}$,

331 which is only marginally larger than the retrieval uncertainty.

333 The vertical profile of haze extinction is the one described above to model the solar flux

334 deposition, scaled to a value of $4.1 \times 10^{-10} \mathrm{~cm}^{-1}$ at $200 \mathrm{~km}$ and $1090 \mathrm{~cm}^{-1}$ as derived by

335 Vinatier et al. (2015) in the $30^{\circ} \mathrm{S}-20^{\circ} \mathrm{N}$ region in $2010-2012$. We added the opacity of the

336 nitrile haze characterized by Anderson and Samuelson (2011). The spectral dependence of

337 this opacity at $15^{\circ} \mathrm{S}$ is given in Fig. 15 of that paper; it peaks at $160 \mathrm{~cm}^{-1}$ and is significant in

338 the range $90-290 \mathrm{~cm}^{-1}$. We used a normal optical depth of 0.0054 at $160 \mathrm{~cm}^{-1}$, as derived by

339 Anderson and Samuelson (2011) at $15^{\circ} \mathrm{S}$, and the associated vertical profile of extinction,

340 having a mass extinction coefficient peaking at $87.5 \mathrm{~km}$ with a full width at half maximum

341 (FWHM) of $18.8 \mathrm{~km}$.

\subsection{Numerical aspects}

344 Starting from the initial temperature profile $T_{0}(p)$, Eq. 1 is integrated through a time-marching

345 scheme, with a constant step $\Delta \xi$ (typically $10^{-3}$ ), $\xi$ being related to the time $t$, Sun distance $d$

346 and heliocentric longitude $\phi$ through the relations (Landau and Lifchitz 1969, Eqs. $(15,10)$

347 and $(15,11))$ :

$348 t=\frac{T_{o r b}}{2 \pi}(\xi-e \sin \xi)$, 
$350 \cos \phi=\frac{\cos \xi-e}{1-e \cos \xi}$

351 where $T_{\text {orb }}$ is Saturn's orbital period, $e$ its orbit eccentricity, $a$ its semi-major axis, with the

352 origin of time and longitude taken at perihelion $(\xi=0, t=0, \phi=0)$. Note that the heliocentric

353 longitude with an origin at northern spring equinox $L_{\mathrm{s}}$ is then equal to $\phi+L_{\mathrm{s}}{ }^{0}, L_{\mathrm{s}}{ }^{0}$ being the

354 value of $L_{\mathrm{s}}$ at perihelion. The solar declination $\delta_{\mathrm{s}}$ is given by:

$355 \sin \delta_{s}=\sin \delta \sin L_{s}$,

356 where $\delta$ is Saturn's obliquity.

358 The time-marching integration is run for long enough so that the influence of the initial 359 profile $T_{0}(p)$ has vanished at the end. At each time step, the diurnally averaged solar flux is 360 derived for the corresponding solar declination and Sun-Saturn distance by integrating over 361 daytime and interpolating as a function of $\cos \left(\theta_{\mathrm{s}}\right)$ from the solar fluxes pre-calculated for 362 zenith angles $\theta_{\mathrm{s}}=0,30,50,60,70,80$ and $85^{\circ}$ (Section 3.1). The thermal flux is calculated 363 from Eq. 5, assuming as a boundary condition a constant surface temperature of $93.5 \mathrm{~K}$ and 364 emissivity of 1.0. Radiative cooling and heating rates are then calculated on the atmospheric 365 pressure grid by differentiation of the solar and thermal fluxes. After adding the adiabatic and 366 advective cooling/heating terms in Eq. 1 to the radiative terms, the variation of temperature $367 \Delta T$ at each level is calculated from Eq. 1 as $\Delta t$ times the sum of these three energy terms, and 368 the process is iterated till the desired date.

\section{Results}


373 Figure 3 shows, at three different pressure levels, the spectral heating rate $h_{\lambda}$, giving the

374 wavelength dependence of the absorbed solar energy (the solar heating rate in Eq. 1 is

$\left.375 h=\int h_{\lambda} d \lambda\right)$. At pressures less than $\sim 5$ mbar, heating is dominated by aerosol absorption of

376 solar radiation at wavelengths below $0.8 \mu \mathrm{m}$, with a peak around $0.4 \mu \mathrm{m}$ at $0.1 \mathrm{mbar}$ and 0.45

$377 \mu \mathrm{m}$ at 1 mbar. At 10 mbar, the strong methane bands between 0.8 and $3.7 \mu \mathrm{m}$ provide

378 substantial additional heating. In this region, methane and aerosol absorption provide

379 comparable contributions to the solar heating while, at deeper levels, methane absorption

380 dominates.

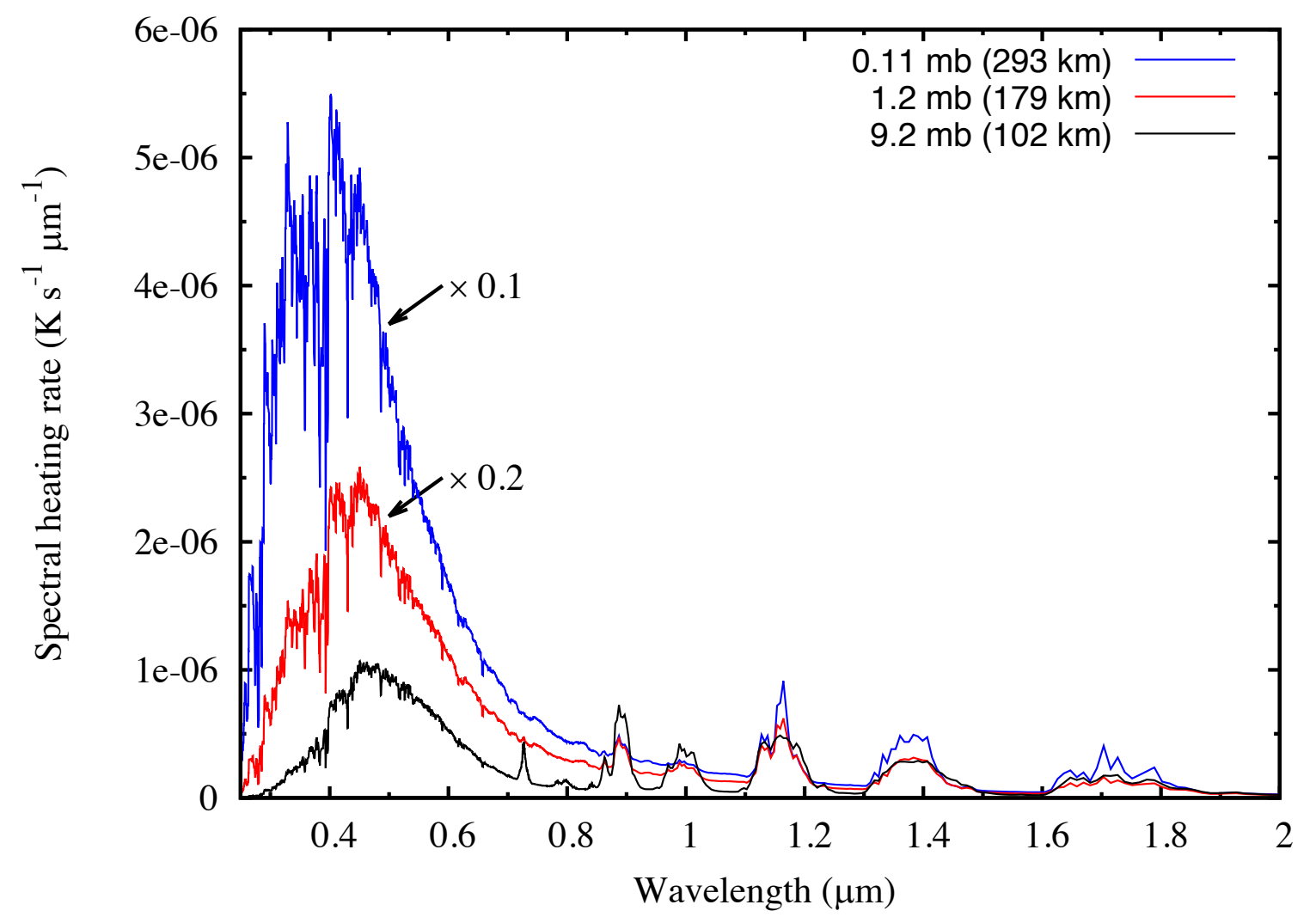

Figure 3: Spectral heating rate $h_{\lambda}$ giving, as a function of wavelength, the contribution per unit wavelength to the day-averaged solar heating rate at three different atmospheric levels. For clarity, $h_{\lambda}$ at $0.11 \mathrm{mbar}$ is multiplied by 0.1 and $h_{\lambda}$ at $1.2 \mathrm{mbar}$ by 0.2 . The solar heating rate is integrated from 0.25 to $3.8 \mu \mathrm{m}$. Insolation conditions correspond to $20^{\circ} \mathrm{S}$ and March 2007. 


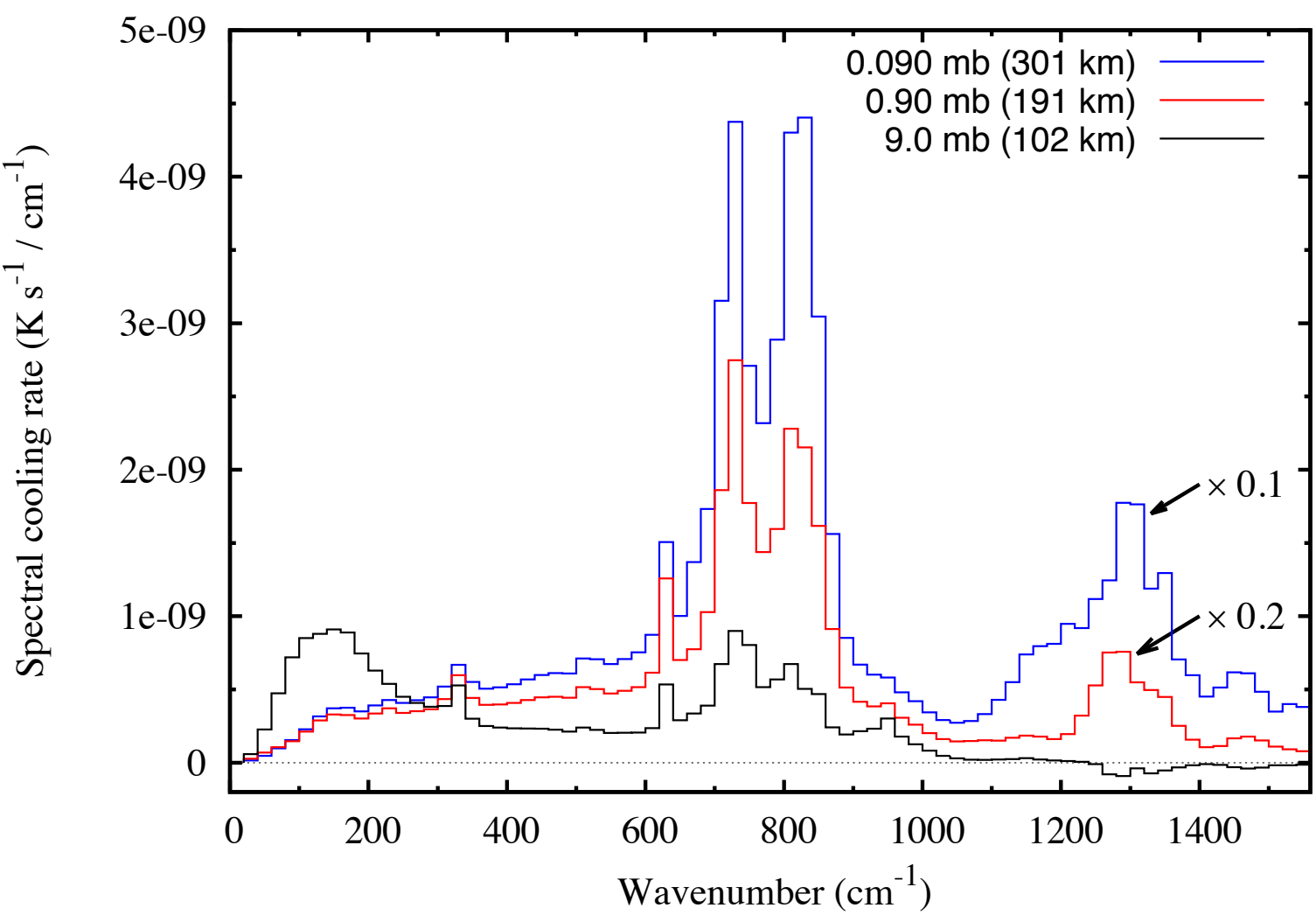

Figure 4: Spectral distribution of the cooling rate $c_{\sigma}$, averaged over $20-\mathrm{cm}^{-1}$ bins, giving, as a function of wavenumber, the contribution to the radiative cooling rate at three different atmospheric levels. For clarity, $c_{\sigma}$ at 0.09 mbar is multiplied by 0.1 and $c_{\sigma}$ at 0.9 mbar by 0.2. The temperature profile pertains to $20^{\circ} \mathrm{S}$ and March 2007.

386 The spectral distribution of the radiative cooling rate $\left(c_{\sigma}\right)$, averaged over 20 -cm-1 intervals, is

387 shown in Fig. 4 at three different levels in the stratosphere. The cooling rate $c$ in Eq. 1 is the 388 integral over wavenumber of $c_{\sigma}: c=\int c_{\sigma} d \sigma$. The most efficient gaseous coolers are ethane $389\left(820 \mathrm{~cm}^{-1}\right)$, acetylene $\left(730 \mathrm{~cm}^{-1}\right)$ and, above the $\sim 5 \mathrm{mbar}$ level, methane $\left(1300 \mathrm{~cm}^{-1}\right)$. Note

390 that at (and below) the 9-mbar level, the methane band heats the atmosphere from above.

391 Substantial cooling in the stratosphere arises from the continuous aerosol opacity. As noted

392 earlier by Tomasko et al. (2008b), the cooling effects of gas and aerosol emission are of the

393 same order. Note, in the 9-mbar cooling rate spectrum, the contribution from the nitrile haze 394 peaking at $160 \mathrm{~cm}^{-1}$ (Anderson and Samuelson 2011). 
396 In Fig. 5, we show the heating and cooling rate profiles calculated with our model for the 397 composition and temperature profile derived from CIRS measurements near $20^{\circ} \mathrm{S}$ in March 398 2007. Both profiles steadily decrease with depth, by more than 3 orders of magnitude from 399 the lower mesosphere to the lower troposphere. In the whole region 0.1-5 mbar, best 400 constrained by CIRS measurements in terms of temperature, haze and composition, the 401 heating and cooling rate profiles are remarkably similar, the former being regularly $20-35 \%$ 402 larger than the latter. The difference between them varies between $3 \times 10^{-6} \mathrm{~K} \mathrm{~s}^{-1}$ at $0.1 \mathrm{mbar}$ 403 and $2 \times 10^{-7} \mathrm{~K} \mathrm{~s}^{-1}$ at 5 mbar. As the observed temperature variation around 2007 is less than 1 $404 \mathrm{~K}_{\text {year }}{ }^{-1}$ (Fig. 1), i.e. $<3 \times 10^{-8} \mathrm{~K} \mathrm{~s}^{-1}$, the energy balance equation (Eq. 1) implies that this 405 difference has to be balanced by adiabatic cooling. This leads to upward velocities decreasing 406 from $0.25 \mathrm{~cm} \mathrm{~s}^{-1}$ at 0.1 mbar to $0.09 \mathrm{~cm} \mathrm{~s}^{-1}$ at $1 \mathrm{mbar}$ and $0.014 \mathrm{~cm} \mathrm{~s}^{-1}$ at $5 \mathrm{mbar}$.

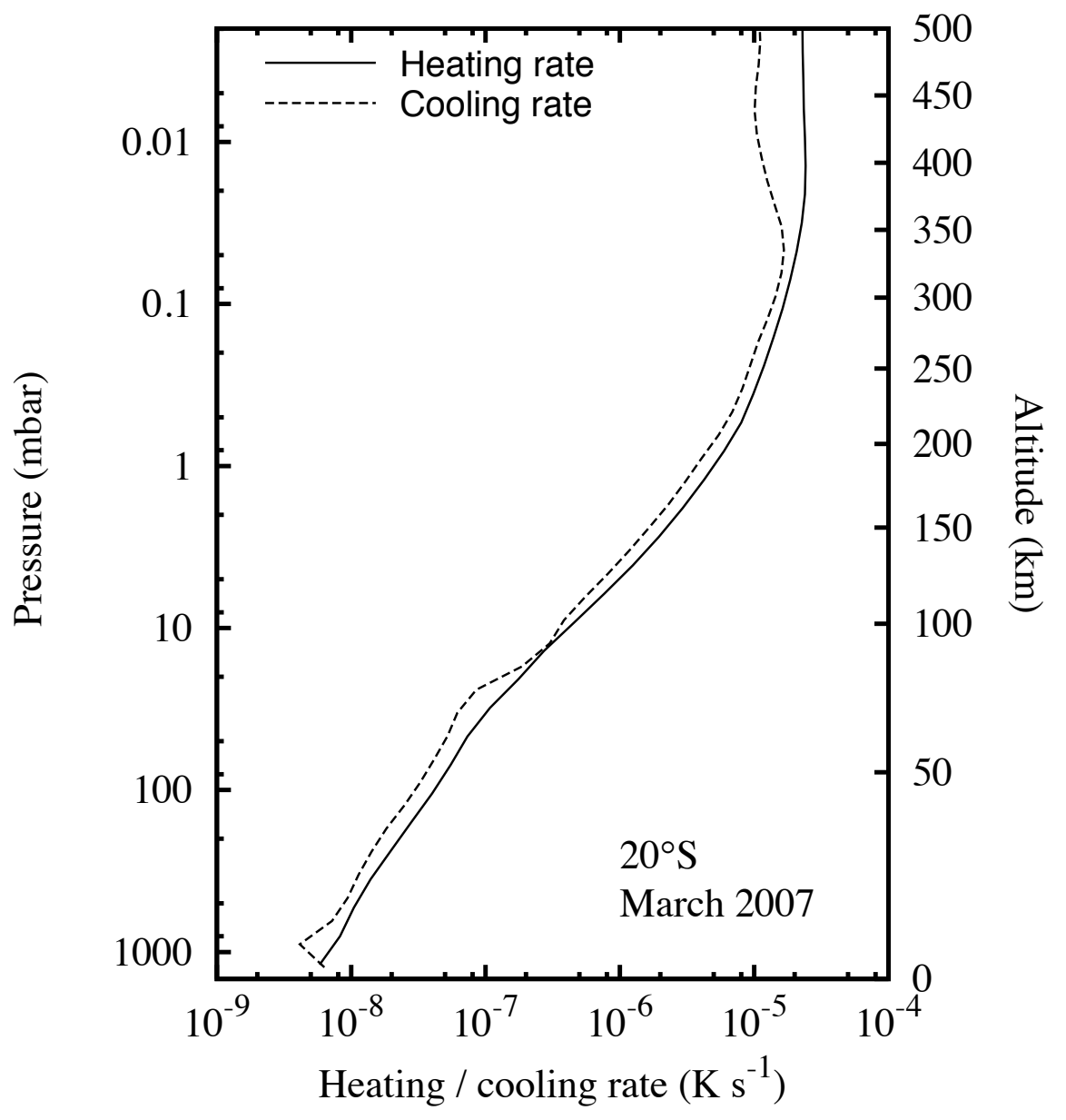


Figure 5: Heating (solid line) and cooling (dashed line) rate profiles calculated with our model using the temperature profile retrieved from CIRS measurements at $20^{\circ} \mathrm{S}$ in March 2007. The heating rate profile corresponds to day-averaged conditions and insolation parameters for March 2007.

408 Around $15 \mathrm{mbar}(85 \mathrm{~km})$, which corresponds to the peak of the nitrile haze, the cooling and

409 heating rates are almost equal. Just below, around $25 \mathrm{mbar}(71 \mathrm{~km})$, the heating rate exceeds

410 the cooling rate by about $70 \%$. This region corresponds to the fall-off of the $\mathrm{C}_{2} \mathrm{H}_{6}$ and $\mathrm{C}_{2} \mathrm{H}_{2}$

411 mixing ratios due to condensation, these two gases being important infrared radiators in the

412 whole stratosphere. Below the 50-mbar level, in the troposphere, the heating rate is about

$41340 \%$ larger than the cooling rate. Note that no information from CIRS data is available using

414 the $v_{4} \mathrm{CH}_{4}$ band below the $\sim 10$-mbar level, and the temperature profile then joins the

415 Huygens/HASI in situ measurements at $10^{\circ} \mathrm{S}$ (Fulchignoni et al. 2005). Above the $\sim 0.03-$

416 mbar level, the heating rate is about twice the cooling rate, which would call from Eq. 1 for an

417 upward velocity $w$ of about $1.5 \mathrm{~cm} \mathrm{~s}^{-1}$. However, this estimation is very uncertain due to the

418 poorly known haze density in this region.

\subsection{Radiative relaxation times}

421 Evaluation of radiative time constants is important to investigate the response of Titan's

422 atmosphere to various perturbations, such as the diurnal and seasonal cycles of insolation, or

423 atmospheric waves. As discussed by Flasar et al. (2014), the radiative time constant $\left(\tau_{r}\right)$ is the

424 characteristic time for radiatively damping out a small perturbation from the equilibrium state,

425 keeping unchanged all other energy terms such as solar and dynamical heating. From Eq. $1, \tau_{r}$

426 is formally equal to the inverse of the derivative of the cooling rate with respect to

427 temperature: 
$\tau_{r}(z)=1 / \frac{\partial c(z)}{\partial T(z)}$

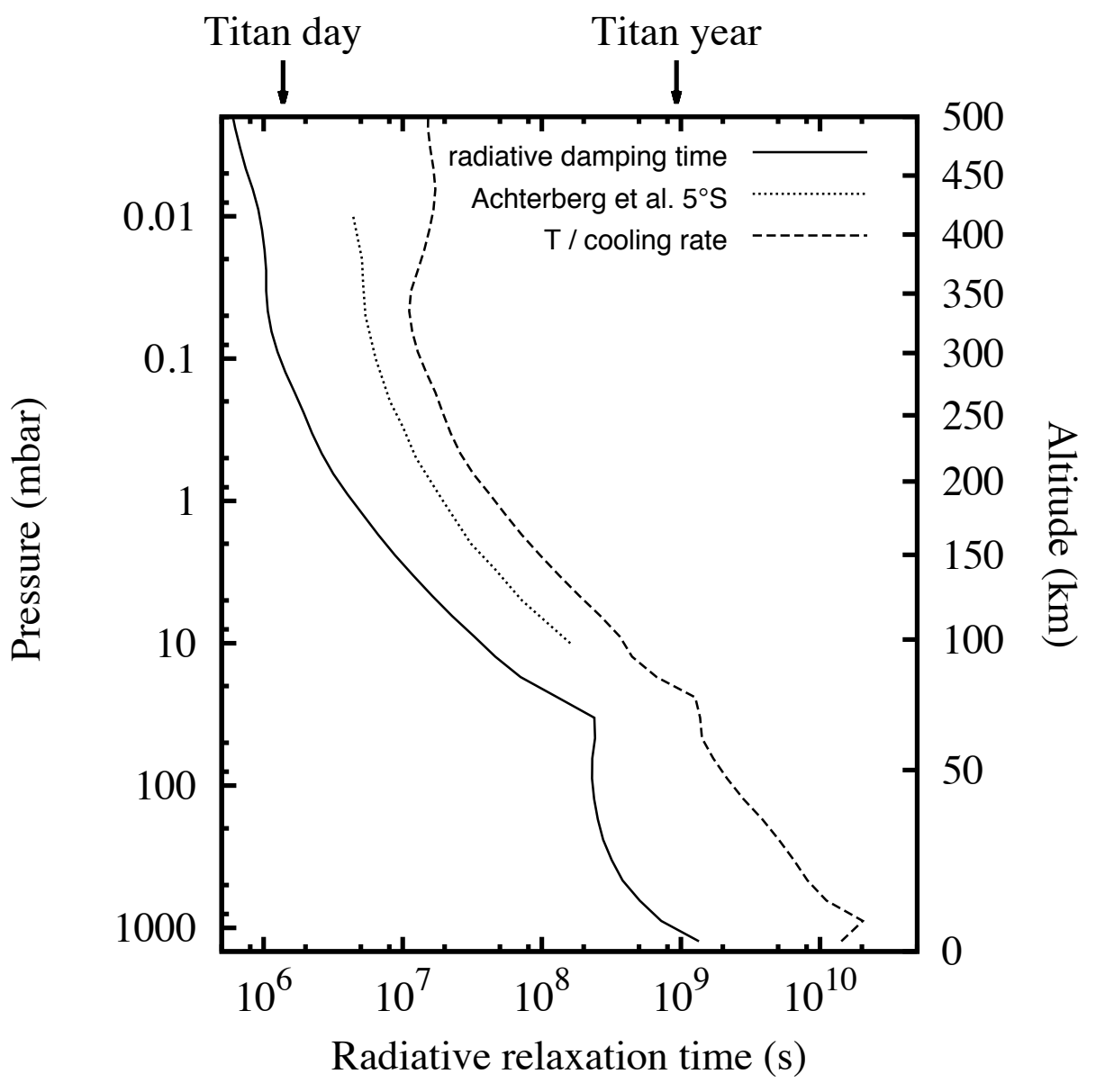

Figure 6: Vertical profiles of radiative relaxation time in Titan's atmosphere. The solid line corresponds to damping out a Gaussian temperature perturbation having a full width at half maximum of one pressure scale height. The dotted line shows the radiative time constant calculated by Achterberg et al. (2011) at $5^{\circ} \mathrm{S}$ using the direct cooling-to-space approximation for the radiative cooling. The dashed line represents the temperature divided by the cooling rate, following the approach of Strobel et al. (2009).

431 However, $c(z)$ in a given layer depends to some extent, through exchange terms, on the

432 temperature outside this layer so that the vertical shape of the assumed perturbation has to be

433 specified. We assumed here a Gaussian perturbation having a FWHM of 1 pressure scale

434 height and centered in a layer $\left[p_{i}: p_{i+1}\right]$. Doing so, $\frac{\partial c(z)}{\partial T(z)}$ can be calculated from Eq. 5 as a 
435 linear combination of terms in the form $\left(A_{i+1, j, k}-A_{i, j, k}\right) \frac{\partial B_{\sigma_{k}}\left[T\left(p_{j}\right)\right]}{\partial T\left(p_{j}\right)}$ where $j$ runs over the 436 perturbed levels with appropriate weighting. The resulting profile of $\tau_{r}$ is shown in Fig. 6 as a 437 solid line.

The radiative time constant readily increases with depth from less than 1 Titan day above the 0.1-mbar level to about 1 Titan year in the lowest layers of the troposphere. In the range 0.1-5 mbar, in which we are mostly interested here, the radiative time constant varies between 0.0015 and 0.02 Titan year, implying that the stratosphere can respond radiatively to the seasonally-varying insolation with negligible time lag. We also made a calculation for a perturbation having a twice larger FWHM (2 scale heights). The resulting time constants are then $\sim 25 \%$ larger in the stratosphere and $\sim 60 \%$ in the troposphere below the 300 -mbar level. at $5^{\circ} \mathrm{S}$ using the direct cooling-to-space approximation and including opacity from $\mathrm{CH}_{4}, \mathrm{C}_{2} \mathrm{H}_{2}$ and $\mathrm{C}_{2} \mathrm{H}_{6}$. Their time constants are 4-5 times larger than those inferred in this work. A factor of $\sim 2$ is likely due to the lack of aerosols and other gases in their calculations and the remaining difference from ignoring the exchange terms, as discussed in Section 5. We also 452 plotted in Fig. 6, the ratio of temperature to cooling rate $(T(z) / c(z))$, which yields a simpler 453 estimate of the radiative timescale. As can be seen from Eq. 1, rather than relevant to damping 454 of a temperature perturbation, this time constant pertains to a case in which the solar heating 455 is turned off (as well as the dynamical term). This is the approach that was used by Strobel et 456 al. (2009) to compute the radiative timescale. Figure 6 shows that, doing so, the radiative 457 damping time is overestimated typically by a factor of 10 . 
460 We first compare the predictions of our seasonal radiative model, with no adiabatic

461 heating/cooling, to the temperature profile retrieved near $6^{\circ} \mathrm{N}$ around northern spring equinox

462 (See Section 2), a region where seasonal variations of insolation are minimal. Figure 7 shows

463 the temperature profile calculated with the three haze profiles of Fig. 2. Temperatures in these

464 models essentially differ above $200 \mathrm{~km}$ due to different assumptions on the vertical profile of

465 aerosol extinction. The warmest profile is associated with the largest aerosol number

466 extinction profile and vice versa, confirming that aerosol heating from scattering and

467 absorption of solar radiation dominates over their cooling effect due to thermal emission. The

468 difference between the extreme profiles increases from $7 \mathrm{~K}$ at 0.1 mbar to $20 \mathrm{~K}$ at $0.001 \mathrm{mbar}$.

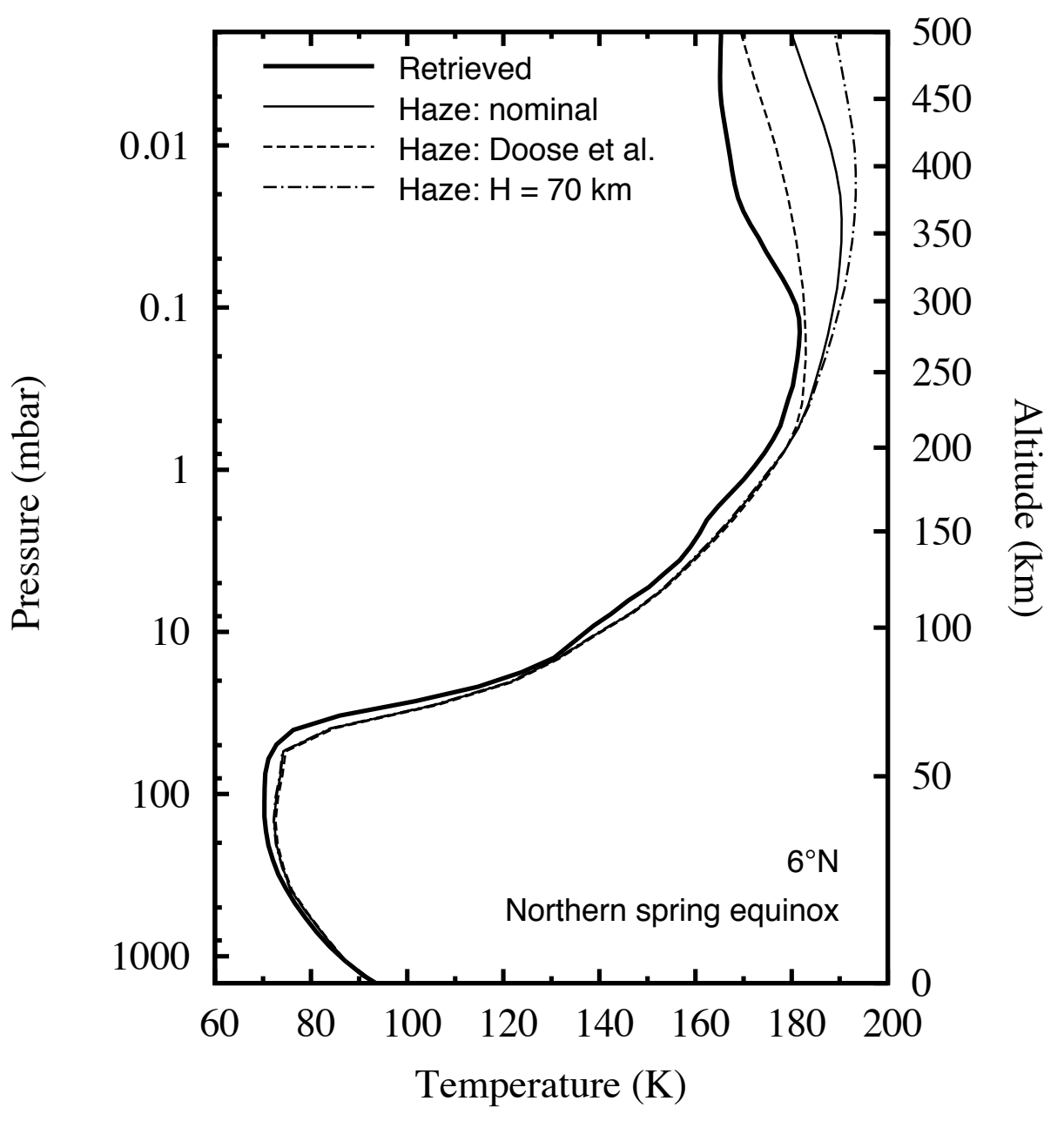

Figure 7: Comparison of a temperature profile retrieved from Cassini/CIRS measurements around $6^{\circ} \mathrm{N}$ and northern spring equinox (thick solid line) with radiative model profiles calculated for the three haze models in Fig. 2. No dynamical term is included in the energy equation. 
471 Whatever haze profile is used, the radiative model profile is warmer than the retrieved one at 472 all levels except around $15 \mathrm{mbar}(85 \mathrm{~km})$. Note that below $5 \mathrm{mbar}$, the profile is not 473 constrained by Cassini/CIRS measurements but essentially represents the Huygens/HASI 474 profile $\left(10^{\circ} \mathrm{S}\right.$, January 2005$)$. To bring the calculated and observed profiles closer, it is 475 necessary to add adiabatic cooling. For our nominal haze model, we find that a constant 476 vertical velocity, expressed in pressure coordinates $\left(\frac{d P}{d t}=-\rho g w\right.$, where $\rho$ is the atmospheric 477 density), of $\approx-1.3$ Pa per Titan day up to the 0.6-mbar level, slightly decreasing in amplitude 478 at higher altitudes to reach $\approx-1.0 \mathrm{~Pa}$ per Titan at $0.02 \mathrm{mbar}$, allows us to reproduce fairly well 479 the observed profile from the 0.05 -mbar level down to the troposphere (Fig. 8, dashed line). 480 The largest discrepancy occurs around 15 mbar, where the model profile is $5 \mathrm{~K}$ colder than 481 the Huygens profile. 


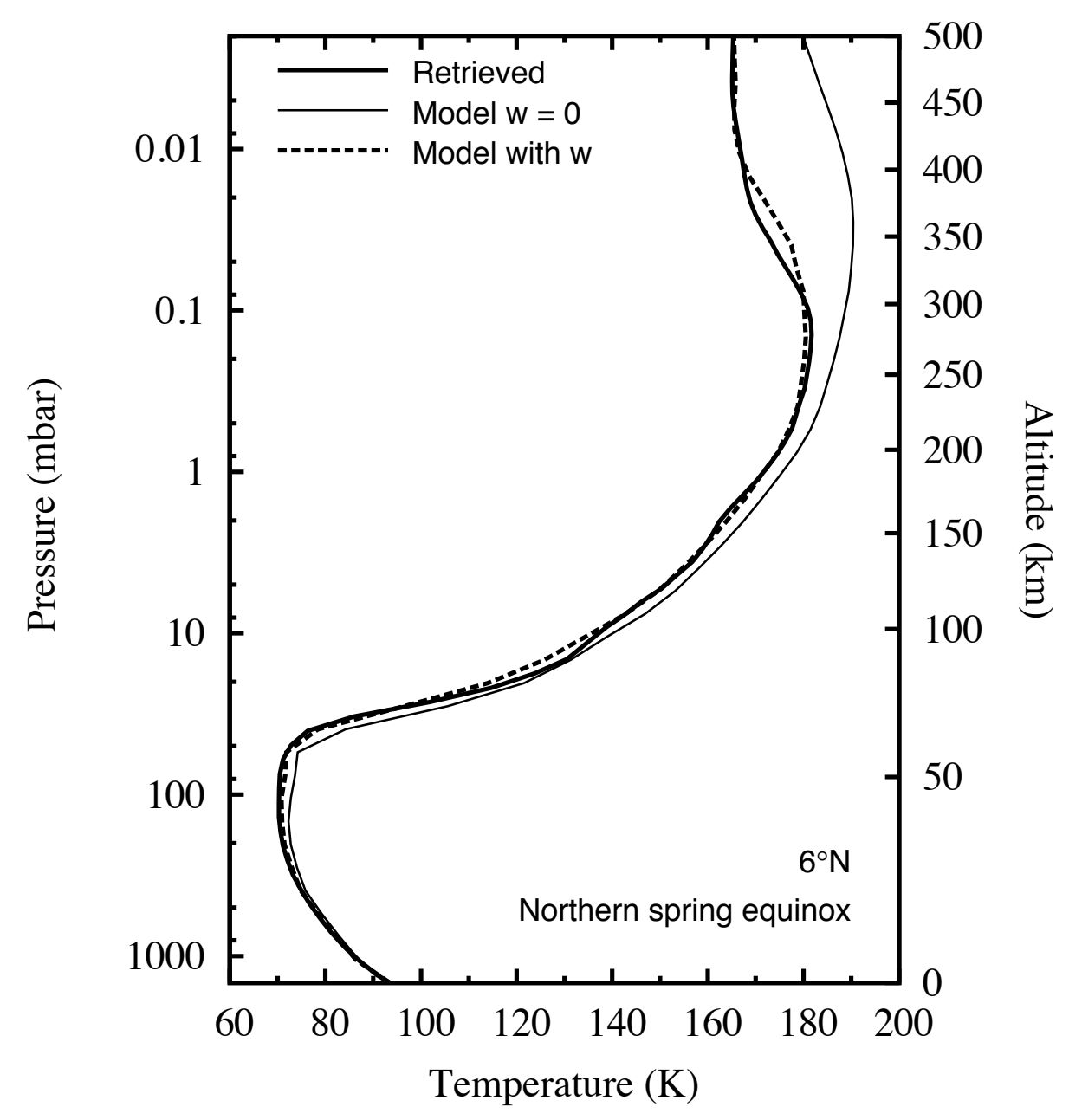

Figure 8: Comparison of a temperature profile retrieved from Cassini/CIRS measurements around $6^{\circ} \mathrm{N}$ and northern spring equinox (thick solid line) with two radiative model profiles using the nominal haze model. The thin solid line shows the case with no additional adiabatic heating (same as in Fig. 7). The dashed line shows a model with a constant velocity below the $0.6-\mathrm{mbar}$ level, when expressed in pressure coordinates, equal to $-1.3 \mathrm{~Pa} /$ Titan day, decreasing to $-1.0 \mathrm{~Pa} /$ Titan day at $0.02 \mathrm{mbar}$ and $-0.5 \mathrm{~Pa} / \mathrm{Titan}$ day at 0.01 mbar (Fig. 9).

484 This velocity profile corresponds to an upward vertical velocity $w$ in the range $0.03-0.05 \mathrm{~cm}$

$485 \mathrm{~s}^{-1}$ at $1 \mathrm{mbar}$, taking into account a $1 \mathrm{~K}$ uncertainty (Fig. 9), which corresponds to the

486 dispersion in our $6^{\circ} \mathrm{N}$ temperature profile selection (Section 2$) . w$ varies as $(\rho g)^{-1}$ below the

487 0.6-mbar level and as $(\rho g)^{-0.92}$ in the 0.6-0.02 mbar range. At higher levels, the velocity is set

488 so that it varies as $(\rho g)^{-0.1}$ from 0.02 to 0.01 mbar, and as $(\rho g)^{0.35}$ above (Fig. 9). The

489 approximate constancy of $\frac{d P}{d t}$ below the 0.02-mbar level suggests only weak divergence of 
490 the upward flow in the stratosphere, while the decrease of $\frac{d P}{d t}$ at higher levels implies stronger

491 divergence, i.e. horizontal poleward motion. However, this conclusion is not firm due

492 uncertainties in the haze profile. It still holds if we use the upper limit for the haze density (" $\mathrm{H}$

$493=70 \mathrm{~km}$ " case) but not for the lower limit (Doose et al. profile), in which case a strong

494 decrease of $\frac{d P}{d t}$ above the $\approx 1$ mbar level is required to reproduce the $6^{\circ} \mathrm{N}$ temperature profile

495 (Fig. 9).
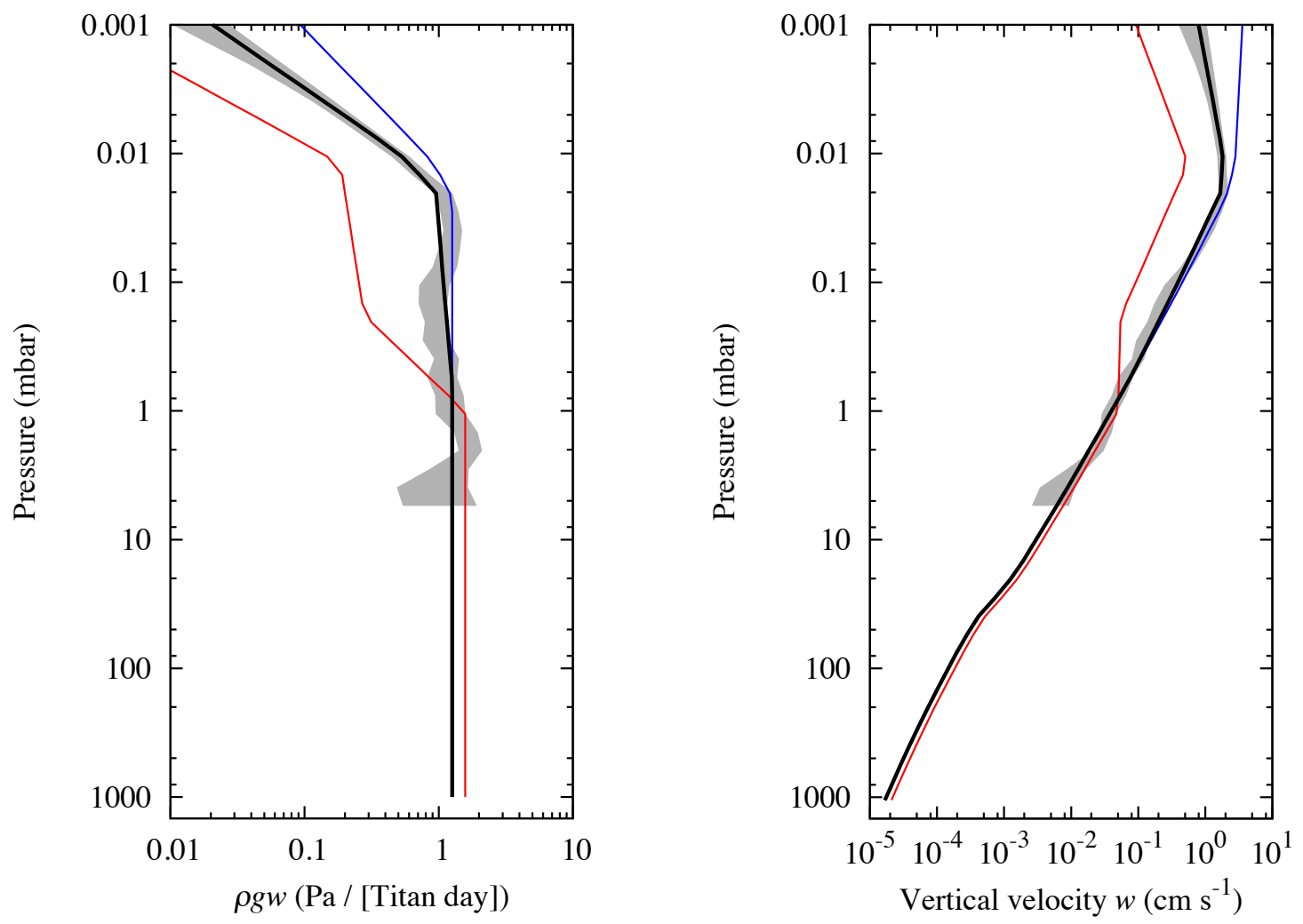

496

Figure 9: Upward velocity profile used to model the temperature profile retrieved at $6^{\circ} \mathrm{N}$ around equinox, given in pressure units per Titan day (left panel) and in $\mathrm{cm} \mathrm{s}^{-1}$ (right panel). The black line corresponds to the model with the nominal haze profile that yields the best fit of the temperature profile (Fig. 8), while the red line corresponds to the Doose et al. profile and the blue line to the " $\mathrm{H}=70 \mathrm{~km}$ " haze profile shown in Fig. 2. The grey area around the best fit velocity profile corresponds to a temperature uncertainty taken as the standard deviation of our $6^{\circ} \mathrm{N}$ temperature profile selection given in Section 2. 
We first investigate here the variations of temperatures at 1 mbar derived from Cassini/CIRS

500 in the equatorial region from 2004 to 2016 (Fig. 1). Figure 10 shows our model predictions

501 using a constant upward velocity of $0.040 \mathrm{~cm} \mathrm{~s}^{-1}$ at $1 \mathrm{mbar}$ as derived above around $6^{\circ} \mathrm{N}$ near

502 northern spring equinox. This model predicts a $\sim 7 \mathrm{~K}$ drop between pre-equinox (2006-2008)

503 and 2016. This variation is due to Saturn's eccentricity of 0.054 , which modulates the

504 distance to the Sun and thus the solar flux striking the Saturn system. A model with zero

505 eccentricity (dashed line in Fig. 10) shows a shallow maximum around mid 2010 and similar

506 temperatures in 2006 and 2016. In contrast, the model accounting for the orbital eccentricity

507 predicts a maximum around 2007, between the perihelion (July 2003) and the equinox

508 (August 2009), followed by a decrease due to the increasing distance with the Sun. In fact,

509 while the temperatures after 2012 are then correctly reproduced by this model, the contrast

510 between pre-equinox and $2016(\sim 7 \mathrm{~K})$ is even larger than the observed value of $\sim 4 \mathrm{~K}$.

511 Increasing the vertical velocity in the model uniformly shifts the calculated temperatures and

512 does not help to reduce the contrast (Fig. 10). To better reproduce the observations, a

513 modulation of the vertical velocity, i.e. of the adiabatic cooling, is required. We chose to do

514 so by simply adding a sine function of the heliocentric longitude $L_{\mathrm{s}}$ to the velocity, which then

515 becomes:

$516 \quad w(p)=w_{c}(p)+w_{m}(p) \sin L_{s}$

517 At the 1-mbar level, $w_{\mathrm{c}}=0.055 \mathrm{~cm} \mathrm{~s}^{-1}$ and $w_{\mathrm{m}}=0.020 \mathrm{~cm} \mathrm{~s}^{-1}$ allows us to reproduce relatively

518 well the observed variation (red line in Fig. 10). The vertical velocity at 1 mbar then varies

519 seasonally between 0.035 and $0.075 \mathrm{~cm} \mathrm{~s}^{-1}$ but is always positive (upward), meaning

520 dynamical cooling of the equatorial region all year through. Note that the vertical profile of $w_{\mathrm{c}}$

521 used here and shown in Fig. 11 differs somewhat from those used at $6^{\circ} \mathrm{N}$ in Section 4.3

522 because it was adjusted to better match the temperatures at $0.2,0.5,1,2$ and 4 mbar retrieved

523 by Achterberg et al. (in preparation) and shown in Section 4.6. 


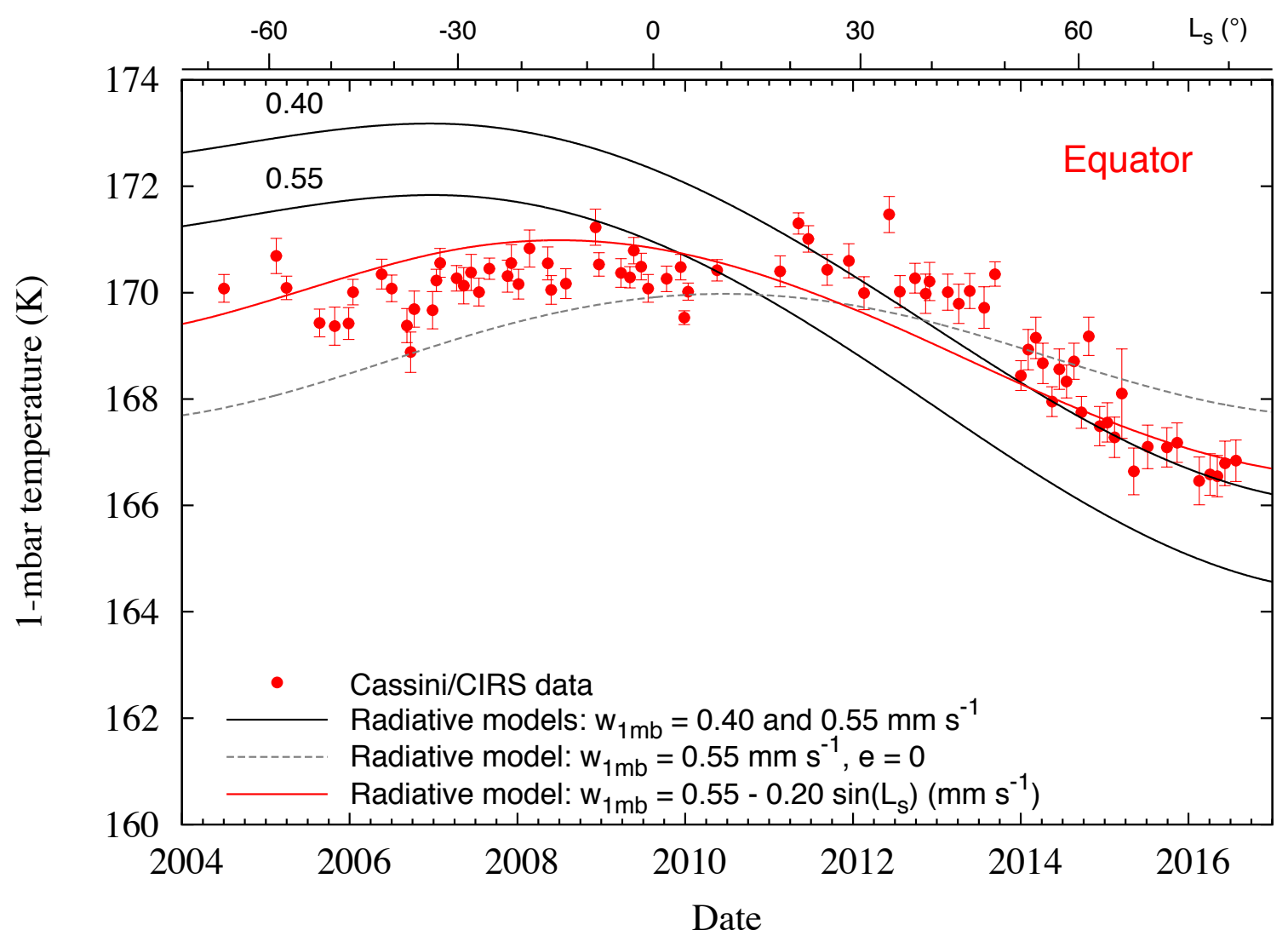

Figure 10: Time variation of 1-mbar temperatures in the equatorial region are compared with predictions from our seasonal radiative model. Solid lines represent models with constant-with-time upward velocity profiles having $w(1 \mathrm{mbar})=0.040$ and $0.055 \mathrm{~cm} \mathrm{~s}^{-1}$. The dashed line represents a model with $w=0.055 \mathrm{~cm} \mathrm{~s}^{-1}$ and the orbital eccentricity set to zero. The red line represents a model with a seasonally-varying vertical velocity profile: $w(1 \mathrm{mbar})=0.055-0.020 \sin \left(L_{\mathrm{s}}\right) \mathrm{cm} \mathrm{s}^{-1}$, where $L_{\mathrm{s}}$ is the heliocentric longitude. 


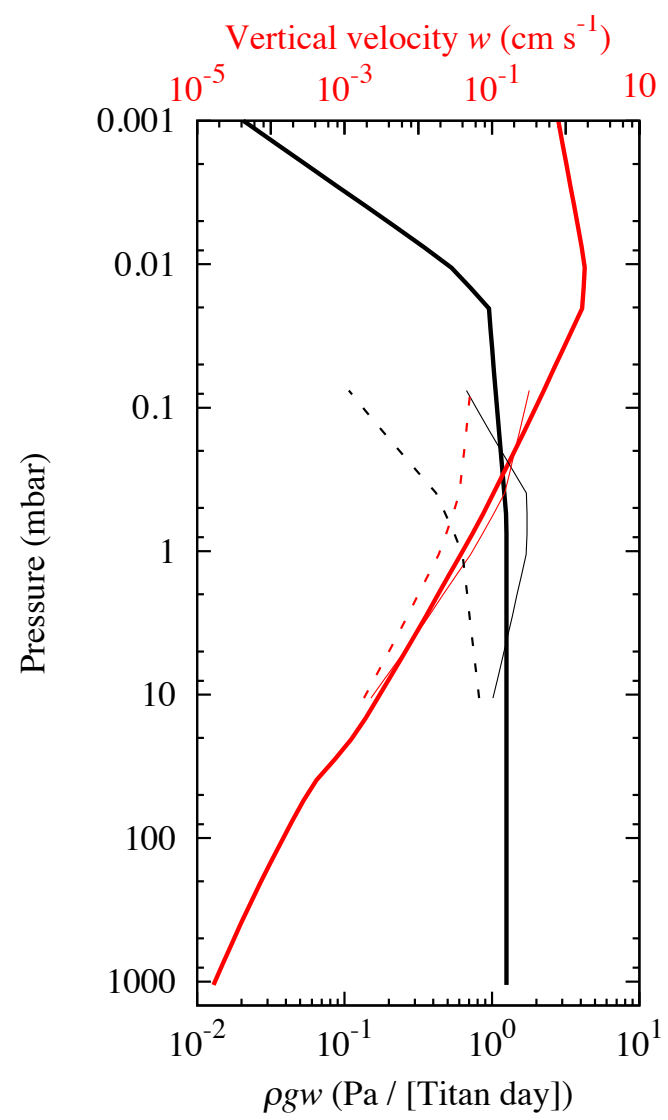

Figure 11: Thick solid lines: upward velocity profile used to model the temperature profile retrieved at $6^{\circ} \mathrm{N}$ around equinox (Fig. 8), given in pressure units per Titan day (black) and in cm s-1 (red). Thin solid lines: year-averaged upward velocity profile $w_{c}$ used to model the seasonal temperature variations at the equator as retrieved by CIRS (Figs. 10 and 15). Thin dashed lines: amplitude of the sine term of this upward velocity profile $w_{m}$. See Eq. 8 . Note that $w_{c}$ and $w_{m}$ are constrained from CIRS observations only from 0.2 to 4 mbar.

We then applied our model to latitudes $30^{\circ} \mathrm{N}$ and $\mathrm{S}$ where seasonal variations of insolation are more pronounced. Figure 12 shows the predicted variations of temperature at 1 mbar over a full Saturnian year (29.46 years), using a constant-with-time upward velocity profile (with $w=0.055 \mathrm{~cm} \mathrm{~s}^{-1}$ at $\left.1 \mathrm{mbar}\right)$. Such a model predicts large variations of temperature as a

532 response to the seasonally-varying insolation with peak-to-peak amplitudes of $33 \mathrm{~K}$ at $30^{\circ} \mathrm{S}$

533 and $19 \mathrm{~K}$ at $30^{\circ} \mathrm{N}$. The amplitudes are stronger in the southern hemisphere than in the northern due to the orbital eccentricity, the perihelion occurring less than a year before southern summer solstice. Clearly, the predicted variations are much stronger than observed 
536 by Cassini since 2004 (Fig. 12). Most noticeable in this comparison is the observed decrease

537 of the 1 -mbar temperature since 2013 at $30^{\circ} \mathrm{N}$, which is at odds with the increase predicted by

538 the radiative model as a result of increasing insolation. This behavior can only be explained

539 by an increase of the dynamical cooling with time at this latitude. More generally, the

540 observed variations at $30^{\circ} \mathrm{N}$ and $\mathrm{S}$ point to dynamics acting to counterbalance the seasonal

541 variations in the solar heating. This means dynamical heating, or reduced cooling, in winter

542 and enhanced dynamical cooling in summer. To model this pattern in a simple way, we

543 proceeded as above and modulated the vertical velocity in the form of Eq. 8. The best fits to

544 the data that we obtained are shown in Figs. 13-14 with the corresponding values of $w_{\mathrm{c}}$ and

$545 w_{\mathrm{m}}$ given in Table 1 . As can be seen, this simple model is able to reproduce the observed

546 variations relatively well.

548 Table 1. Model parameters for the vertical velocity profile ${ }^{1}$

\begin{tabular}{|c|c|c|c|c|c|}
\hline Latitude & $\begin{array}{l}w_{c}(1 \text { mbar }) \\
\left(\mathrm{cm} \mathrm{s}^{-1}\right)\end{array}$ & $\begin{array}{l}w_{m}(1 \mathrm{mbar}) \\
\left(\mathrm{cm} \mathrm{s}^{-1}\right)\end{array}$ & $\begin{array}{l}\text { Pressure range } \\
\text { (mbar) }\end{array}$ & $\alpha_{c}$ & $\alpha_{m}$ \\
\hline Equator & +0.055 & -0.020 & $<0.01$ & -0.40 & -0.40 \\
\hline $30^{\circ} \mathrm{N}$ & +0.063 & +0.075 & $0.01-0.025$ & 0.20 & 0.20 \\
\hline \multirow[t]{3}{*}{$30^{\circ} \mathrm{S}$} & +0.053 & -0.105 & $0.025-0.4$ & 0.45 & 0.30 \\
\hline & & & $0.4-1$ & 1.0 & 0.60 \\
\hline & & & $>1$ & 1.2 & 0.90 \\
\hline
\end{tabular}

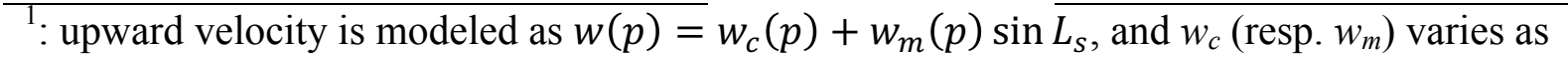

$550(\rho g)^{-\alpha_{c}}\left(\right.$ resp. $\left.(\rho g)^{-\alpha_{m}}\right)$ in a given pressure range. 


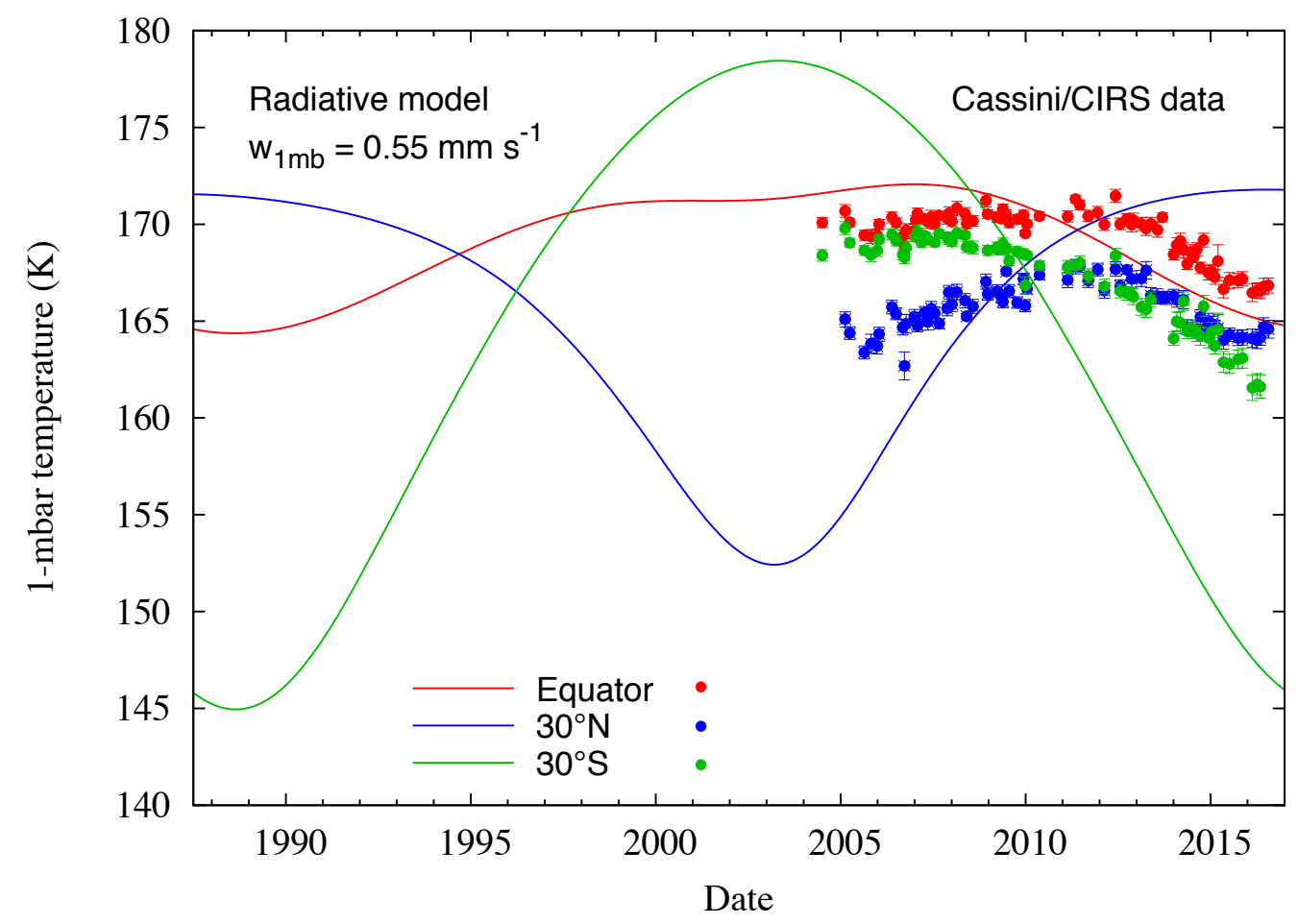

Figure 12: Time variation of 1 -mbar temperatures at $0^{\circ}, 30^{\circ} \mathrm{N}$ and $30^{\circ} \mathrm{S}$ predicted by the seasonal radiative model using a constant-with-time upward velocity profile with $w(1$ mbar) $=0.055 \mathrm{~cm} \mathrm{~s}^{-1}$. Data retrieved from Cassini/CIRS measurements (Fig. 1) are overplotted for comparison.

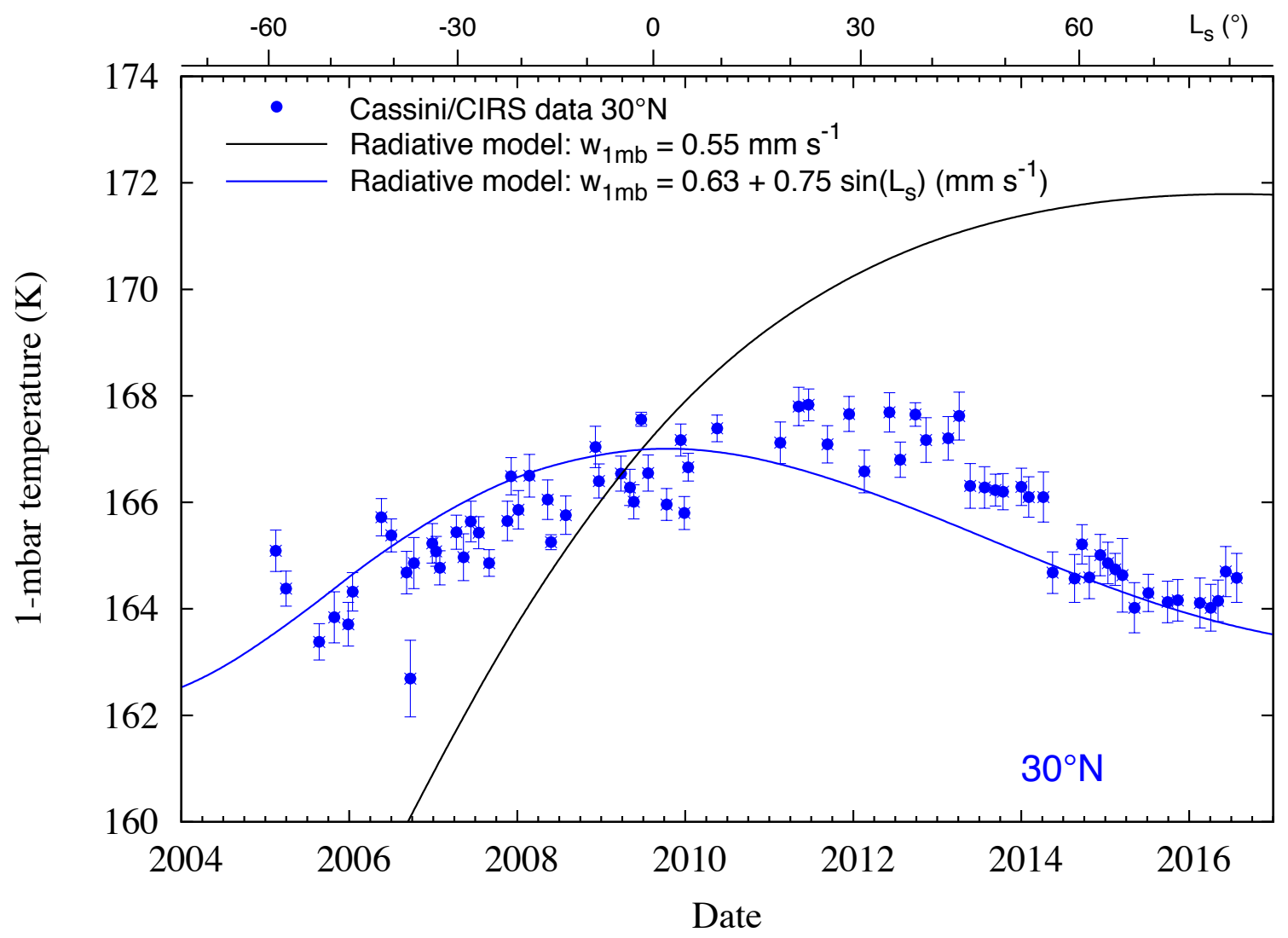


Figure 13: Time variation of $1-\mathrm{mbar}$ temperatures at $30^{\circ} \mathrm{N}$ predicted by the seasonal radiative model are compared with data retrieved from Cassini/CIRS measurements (Fig. 1). Black line: constant-with-time vertical velocity; colored line: vertical velocity varying with solar longitude (see parameters in Table 1).

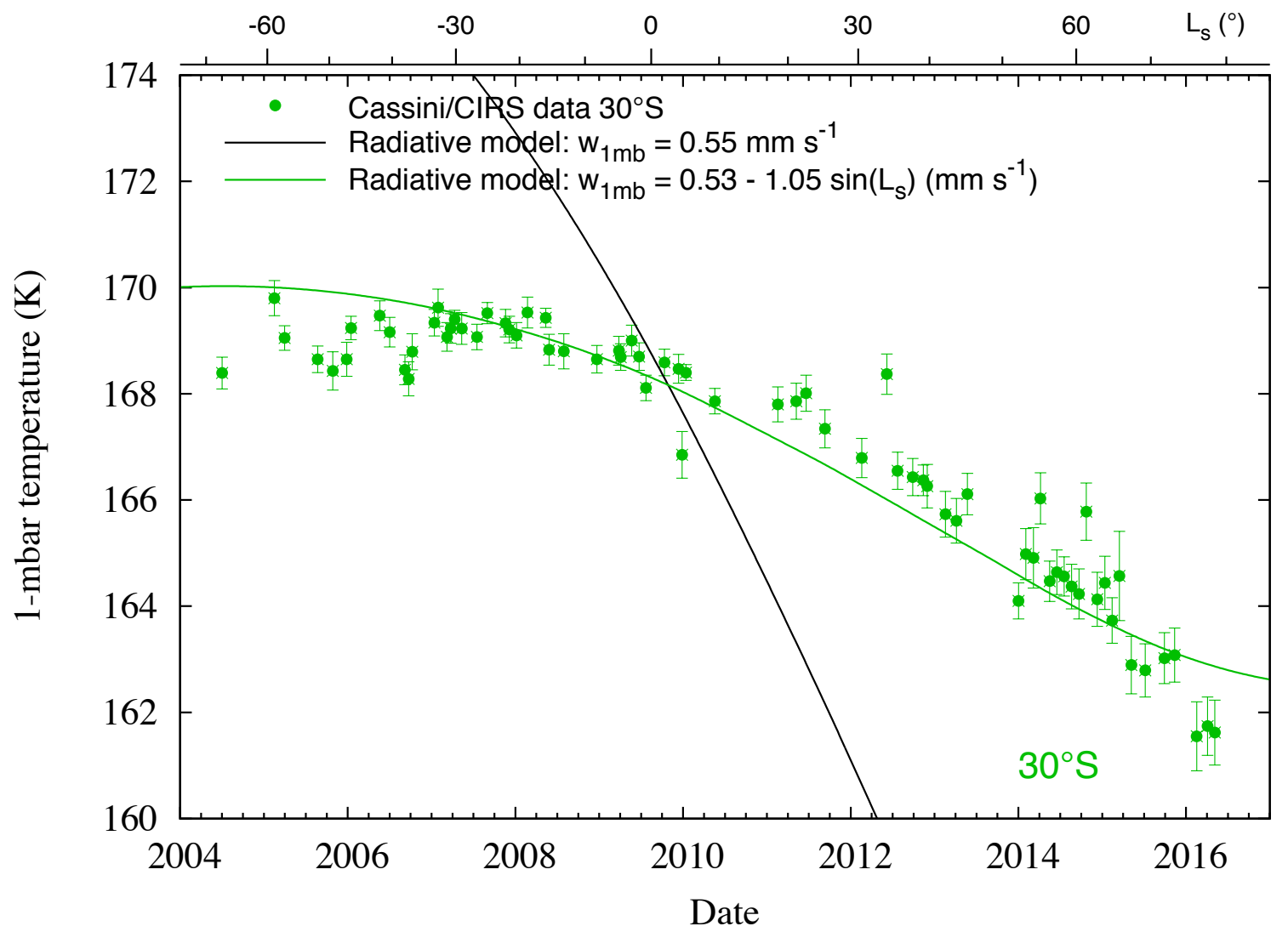

560

Figure 14: Same as Fig. 12 for $30^{\circ} \mathrm{S}$.

\subsection{Seasonal variations of temperature at other levels}

In Section 4.4, we focused on the 1-mbar level, which is the region best constrained by

Cassini/CIRS observations in terms of haze properties and temperature, and we have tuned our model to best reproduce the data at this level. In this section, we extend our modelling up to 0.2 mbar and down to 4 mbar, where temperature information is also available (Fig. 15). To do so, we used a vertical velocity profile $w(p)$ varying as $(\rho g)^{-\alpha}$ in a given pressure range as indicated in Table 1 and shown in Fig. 9 at the equator. As we intend to keep a smooth variation for $w(p)$, we did not try to match exactly the average temperature at a given pressure 
level apart from that at 1 mbar. We then have typical discrepancies of $\pm 3 \mathrm{~K}$ on the average

572 temperatures which may be due to uncertainties in our haze model above $\sim 0.4$ mbar (Fig. 7)

573 and possibly systematic uncertainties in the temperature retrievals below $\sim 2$ mbar. We are

574 therefore more interested here in the seasonal variations of temperature at a given level than

575 in their absolute mean values.

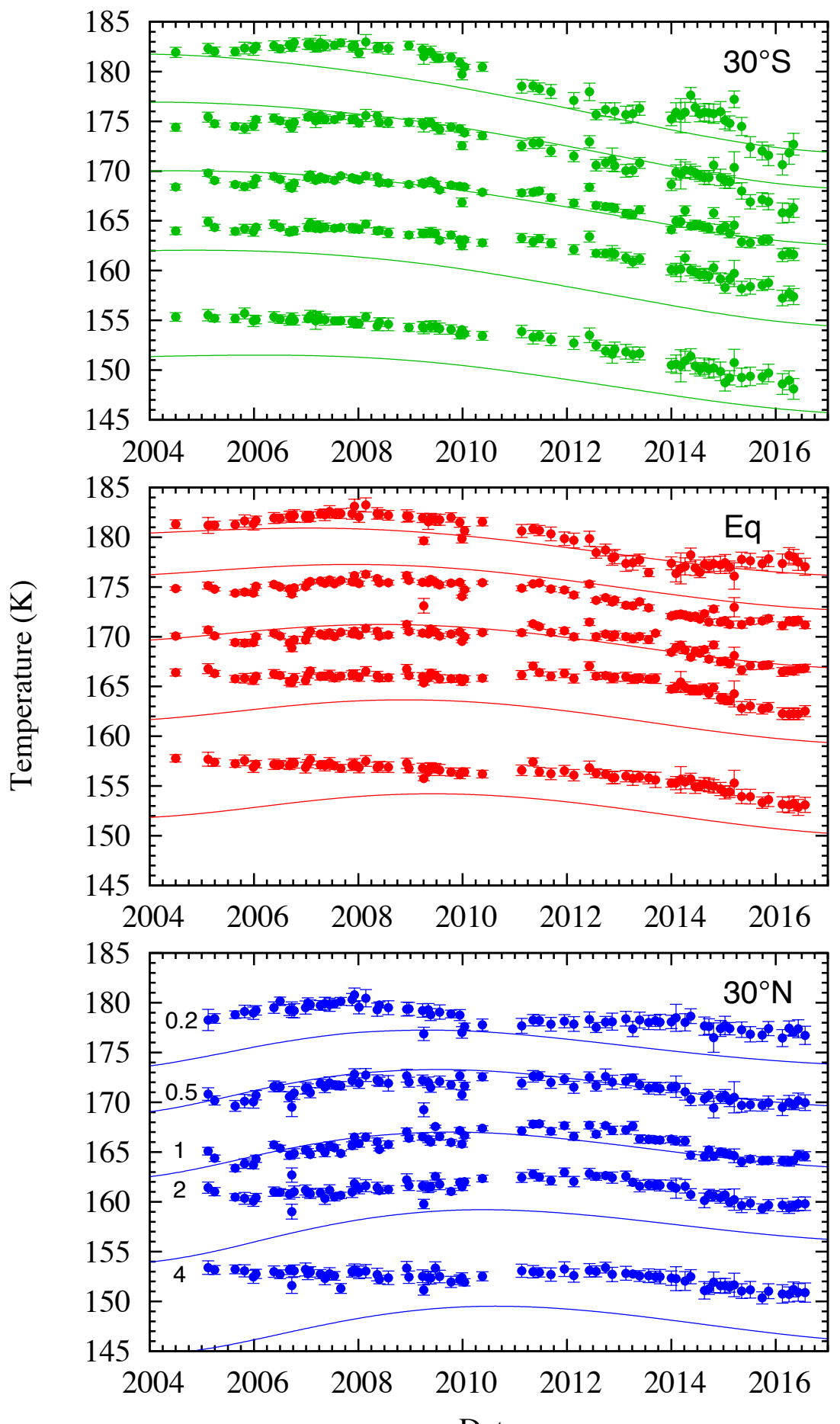

Date 
Figure 15: Time variation of temperatures at $0.2,0.5,1,2$ and 4 mbar predicted by the seasonal radiative model (colored lines) are compared with data retrieved from Cassini/CIRS measurements at $30^{\circ} \mathrm{S}$, equator and $30^{\circ} \mathrm{N}$. In this model, the vertical velocity varies as an affine function of $\sin L_{s}$ (see parameters in Table 1).

581 In Fig. 15, we show the seasonal variations of temperature predicted by our model for

582 different pressure levels at $0,30^{\circ} \mathrm{N}$ and $30^{\circ} \mathrm{S}$. In these "best-fit" models, the vertical variation

583 of the constant and sine components of the velocity profile were chosen to reproduce

584 satisfactorily the observed seasonal variations at the three latitudes simultaneously (only one

585 set of parameters for all latitudes, given in Table 1). On the other hand, the velocity terms $w_{c}$

586 and $w_{m}$ at 1 mbar were adjusted at each latitude to best reproduce the seasonal variations at

587 this level, as discussed in Section 4.4. The CIRS-derived variations are overall fairly well

588 reproduced except noticeably at 4 mbar where the steady decrease of temperature at the

589 equator and at $30^{\circ} \mathrm{N}$ before equinox (2009) is not predicted by the model.

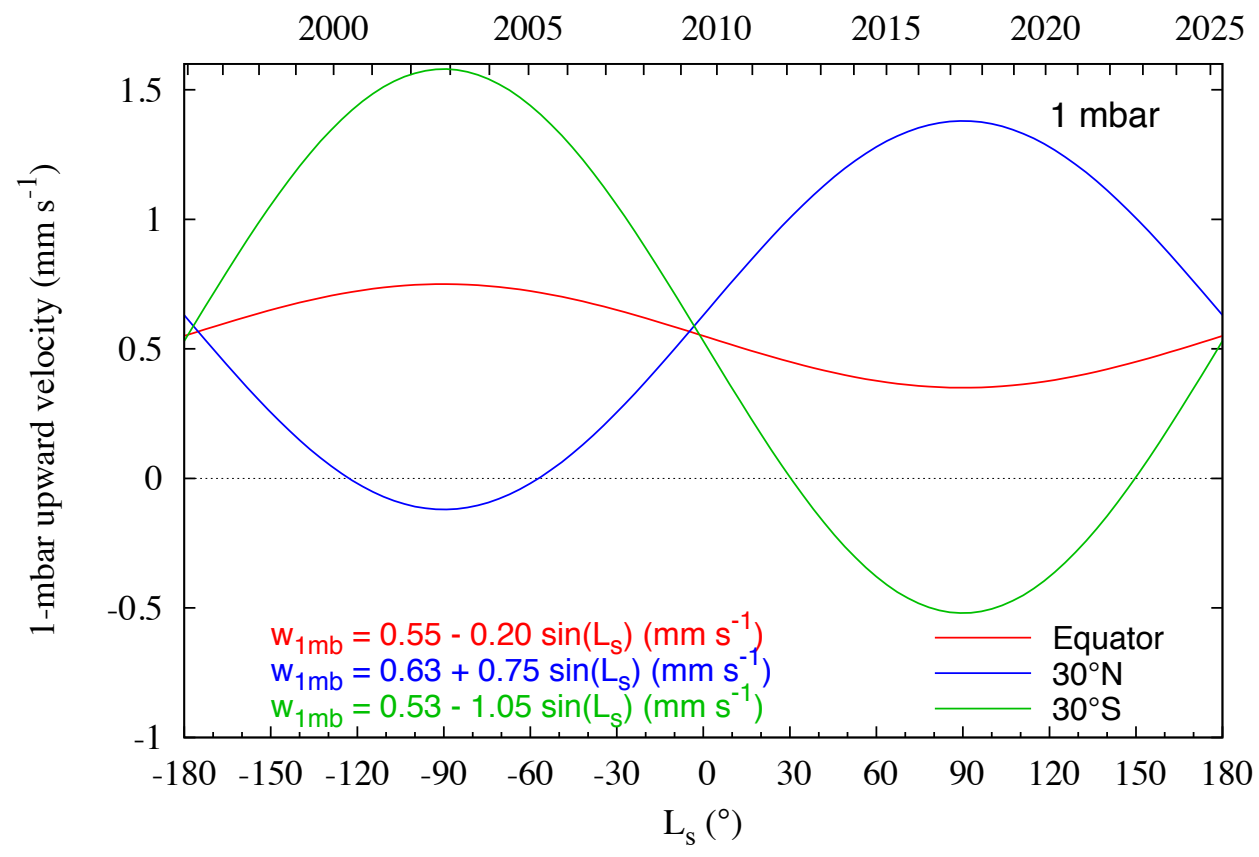

Figure 16: Time variation of the 1-mbar model upward velocity at $0^{\circ}, 30^{\circ} \mathrm{N}$ and $30^{\circ} \mathrm{S}$ as a function of heliocentric longitude (lower scale) and year (upper scale). 
593 The seasonal variation of the model velocity profile $w$ at 1 mbar is shown in Fig. 16 for the

594 three latitudes. The seasonal variation at other pressure levels can be obtained from the 595 parameters in Table 1. The vertical dependence of the two components ( $w_{c}$ and $w_{m}$ in Eq. 8$)$ is 596 illustrated in Fig. 11.

\section{5. Discussion}

\subsection{Heating and cooling rates}

601 Tomasko et al. (2008b) computed the solar heating rate at Huygens probe-landing latitude, 602 averaged over longitude, using the haze model derived from DISR measurements by 603 Tomasko et al. (2008c) and methane absorption coefficients simultaneously derived from 604 DISR measurements (Tomasko et al. 2008a). The solar heating rates we derived at $20^{\circ} \mathrm{S}$ in 605 March 2007 do not differ from those of Tomasko et al. by more than $\pm 25 \%$ in the range 10 $606170 \mathrm{~km}$. The largest differences occur at 10 and $170 \mathrm{~km}$ where our heating rate is some $20 \%$ 607 larger and near $90 \mathrm{~km}$ where our heating rate is $25 \%$ smaller. We regard these differences as 608 acceptable given the difference in the haze model, which was recently updated by Doose et al. 609 (2016), and in the methane absorption, which we calculate using the ab initio TheoReTS 610 database (Rey et al. 2017).

612 Cooling rates corresponding to the temperature, gas and haze profiles derived from Huygens 613 and Cassini/CIRS data were also calculated by Tomasko et al. (2008b). Our cooling rate 614 profile agree with theirs to within $\pm 20 \%$ in the whole altitude range $10-400 \mathrm{~km}$, with the 615 maximum discrepancy occurring near $10 \mathrm{~km}$ (ours is $20 \%$ smaller) and $70 \mathrm{~km}$ (ours is $20 \%$ 616 larger). 
618 Comparing the heating and cooling rates calculated at the Huygens landing site, Tomasko et

619 al. (2008b) concluded that the former exceeds the latter by a maximum of $0.5 \mathrm{~K}$ per Titan day

620 near $120-130 \mathrm{~km}$ and that the excess decreases strongly below and above this altitude. At 125

$621 \mathrm{~km}$, we derive $0.4 \mathrm{~K} /$ Titan day, in good agreement with Tomasko et al. Below this altitude,

622 we also find that the net radiative heating minus cooling rate decreases rapidly, e.g. $0.07 \mathrm{~K} /$

623 Titan day at $70 \mathrm{~km}$ and $0.02 \mathrm{~K} /$ Titan day at $50 \mathrm{~km}$, quite similar to Tomasko et al.'s results.

624 On the other hand, we do not infer any decrease of this net radiative heating minus cooling

625 above $120-130 \mathrm{~km}$, but instead a regular increase up to 3 to $4 \mathrm{~K}$ per Titan day at 250-300 km.

626 We note however that the decrease at higher altitudes invoked by Tomasko et al. is very

627 uncertain given the large uncertainties in their haze model above $140 \mathrm{~km}$, the altitude where

628 the Huygens probe began measurements. Our haze model is also uncertain above $\sim 200 \mathrm{~km}$,

629 where the net radiative heating minus cooling rate reaches $\sim 2 \mathrm{~K}$ per Titan day.

630

631 We can also compare our calculations with the General Circulation Model (GCM) of

632 Lebonnois et al. (2012, Fig. 10). At $20^{\circ} \mathrm{S}$, their annual average of the solar heating rate is

633 almost twice as large as ours at $90-100 \mathrm{~km}, 50 \%$ larger at $60 \mathrm{~km}$ and $30 \%$ larger at $50 \mathrm{~km}$.

634 These differences likely originate from differences in the haze model, that in Lebonnois et

635 al.'s GCM being coupled with the 3-dimensional circulation and not imposed from

636 observational constraints. More significant for our model comparison may be the ratio of

637 dynamical cooling to total (radiative plus dynamical) cooling, also equal to radiative solar

638 heating on an annual average basis. At $6^{\circ} \mathrm{N}$, where seasonal modulation is minimum, this ratio

639 can be determined from Fig. 10 of Lebonnois et al. at 85 and $66 \mathrm{~km}$ as approximately $18 \%$

640 and $25 \%$ respectively. Using the vertical velocity of $1.1 \mathrm{~Pa} / \mathrm{Titan}$ day that we inferred in

641 Section 4.3 at this latitude and the yearly-averaged solar heating rate from our model, we

642 obtain ratios of $17 \%$ at $85 \mathrm{~km}$ and $28 \%$ at $66 \mathrm{~km}$, in excellent agreement with Lebonnois et 
643 al.'s GCM outputs. Lebonnois et al. (2012) do not provide information to compare results

644 above $100 \mathrm{~km}$.

645

646

\subsection{Radiative relaxation times}

647 In Section 4.2, we calculated the radiative time constant $\left(\tau_{R}\right)$ corresponding to damping a

648 temperature perturbation relative to the radiative equilibrium state, having a FWHM of one

649 pressure scale height (Fig. 6). This time constant depends somewhat on the width of the

650 temperature perturbation and doubling the width increases its value by $25 \%$ in the

651 stratosphere. Strobel et al. (2009) provided another estimate of a radiative timescale by

652 dividing temperature by the cooling rate, which actually corresponds to the time decay of

653 temperature when the solar heating is turned off starting from the radiative equilibrium state.

654 While this timescale may be appropriate to investigate diurnal variations of temperature, it

655 strongly overestimates the time constant associated with the damping of small temperature

656 perturbations around the radiative equilibrium state such as those caused by gravity waves or

657 moderate seasonal variations of insolation. Figure 6 shows that our time constant $\tau_{R}$ is about a

658 factor of 10 smaller than the timescale of Strobel et al., which is important to evaluate

659 correctly the response of the atmosphere to e.g. seasonal forcing or planetary waves. For

660 example, our calculation indicates that $\tau_{R}$ exceeds 1 Titan year only in the first $7 \mathrm{~km}$ of

661 Titan's atmosphere while Strobel et al.'s estimation would predict that it happens at all

662 altitudes below $76 \mathrm{~km}$. In the upper troposphere and tropopause region, $30-60 \mathrm{~km}, \tau_{R}$ is 0.25

663 to 0.3 Titan year, which allows for non-negligible seasonal variations of temperature in

664 contrast to expectations using Strobel et al.'s simpler formulation. In the stratosphere, above

$665100 \mathrm{~km}(p<10 \mathrm{mbar}), \tau_{R}$ is less than 0.03 Titan year (25 Titan days), so that significant

666 seasonal temperature variations with almost no phase lag are expected.

667 
As discussed by Flasar et al. (2014), the radiative time constant $\tau_{R}$, as we define it here, can

669 be formally written as $\left(\frac{\partial c(z)}{\partial T(z)}\right)^{-1}$ while Strobel et al. simply used $\left(\frac{c(z)}{T(z)}\right)^{-1}$. Using the cooling-

670 to-space approximation and assuming that thermal cooling mostly occurs between the $\mathrm{C}_{2} \mathrm{H}_{2}$

671 band at $729 \mathrm{~cm}^{-1}$ and the $\mathrm{CH}_{4}$ band at $1305 \mathrm{~cm}^{-1}$, Flasar et al. estimated that the difference

672 between the two estimations in the stratosphere could amount to a factor between 6 and 11,

673 roughly in agreement with the factor of $\sim 10$ we find.

674

675 The cooling-to-space approximation was used by Achterberg et al. (2011) to estimate the 676 radiative relaxation time at $5^{\circ} \mathrm{S}$ in the stratosphere. While their results are a factor of 2 to 3 677 smaller than $\frac{T(z)}{c(z)}$, they are still 4-5 times larger than our $\tau_{R}$ (Fig. 6). Achterberg et al. only 678 considered opacity from $\mathrm{C}_{2} \mathrm{H}_{6}, \mathrm{C}_{2} \mathrm{H}_{2}$ and $\mathrm{CH}_{4}$ and, based on Tomasko et al.'s (2008b)

679 calculations, we estimate that the omission of other gases, and more importantly of haze, 680 could explain a factor of 2 difference with our $\tau_{R}$. The rest is at least partly due to the use of 681 the cooling-to-space approximation which differs from our calculations where the perturbed 682 layer, only one scale height thick, can also significantly cool by emitting to other atmospheric layers in addition to space.

\subsection{Energy balance and adiabatic cooling}

686 Comparing the radiative heating and cooling rates at $20^{\circ} \mathrm{S}$ in March 2007 (Section 4.1), we 687 found that the former exceeds the latter by $20-35 \%$ in the range $0.1-5$ mbar. Given that the 688 actual temperature variation is at least an order of magnitude smaller than the radiative cooling rate, conservation of energy implies an additional source of cooling to balance the

690 heating. The vertical upward velocity required to produce this cooling is about $0.09 \mathrm{~cm} \mathrm{~s}^{-1}$ at 1 691 mbar and varies approximately as $(\rho g)^{-1}$ from 1 to 5 mbar and as $(\rho g)^{-0.45}$ from 1 to 0.1 mbar. 
693 Similarly, around $6^{\circ} \mathrm{N}$, a latitude least sensitive to seasonal effects, the radiative heating

694 exceeds the radiative cooling around equinox at all altitudes. As a result, the radiative solution

695 for the temperature profile is warmer than the observed one at all levels (Section 4.3, Fig. 7).

696 An exception is the region around $85-90 \mathrm{~km}$, where the two profiles exhibit the same

697 temperature with equal heating and cooling rates. This is due to the presence of the nitrile

698 haze parametrized by Anderson and Samuelson (2011) which increases the cooling rate by

$699 \sim 30 \%$ at this altitude. The radiative equilibrium solution without this nitrile haze would be

700 about $4 \mathrm{~K}$ warmer at $85-90 \mathrm{~km}$ where it peaks according to Anderson and Samuelson. Above

$701200 \mathrm{~km}$, the uncertainty in the model temperature profile due the haze profile increases with

702 height (Fig. 7). The difference between models using the lower and upper limits we fixed on

703 the haze profile (Fig. 2) reaches $\sim 3 \mathrm{~K}$ at $250 \mathrm{~km}(0.25 \mathrm{mbar}), 7 \mathrm{~K}$ at $300 \mathrm{~km}(0.09 \mathrm{mbar})$,

$704 \sim 13 \mathrm{~K}$ at $350 \mathrm{~km}(0.035$ mbar $)$ and $\sim 15 \mathrm{~K}$ at $400 \mathrm{~km}(0.013$ mbar $)$. The coldest profile

705 corresponds to the model of Doose et al. (2016) which has less aerosol density than the two

706 other models. In fact, aerosol opacity provides both atmospheric cooling at long wavelengths

707 and heating in the UV to near-infrared range, but the contribution to the heating dominates

708 over that to the cooling.

710 To bring the model in general agreement with the Cassini/Huygens-derived profile at $6^{\circ} \mathrm{N}$, we

711 used an upward velocity profile $w$ having $0.04 \mathrm{~cm} \mathrm{~s}^{-1}$ at $1 \mathrm{mbar}$, varying as $(\rho g)^{-1}$ below the

$7120.6-\mathrm{mbar}$ level down to the troposphere, and as $(\rho g)^{-0.92}$ between 0.6 and $0.02 \mathrm{mbar}$ (for our

713 nominal haze model). The largest discrepancy takes place at altitudes of Anderson and

714 Samuelson's (2011) nitrile haze ( $85-90 \mathrm{~km}, \sim 15 \mathrm{mbar})$ where the model is $\sim 5 \mathrm{~K}$ colder than

715 measured by Huygens/HASI at $10^{\circ} \mathrm{S}$ in 2005 . This velocity profile corresponds to $\sim-1$ to -1.5

716 Pa per Titan day below the 0.02-mbar level $(375 \mathrm{~km})$, implying, from conservation of mass,

717 no strong divergence of the vertical flux, i.e. weak variations of the latitudinal flow (black 
718 lines in Fig. 9). On the other hand, above the 0.02-mbar level, the vertical mass flux in this

model is no longer approximately conserved and diverges horizontally in latitude. Note however that the velocity profile above $\sim 220 \mathrm{~km}(0.5 \mathrm{mbar})$ is relatively uncertain due to uncertainties in the haze opacity profile. Using our lower limit for the haze density profile a significant divergence of the vertical mass flow is needed at much lower levels, $\sim 1$ mbar (Fig. 9). Also, although the nominal velocity profile allows us to reproduce generally well the temperature profile retrieved at $6^{\circ} \mathrm{N}$, discrepancies of a few degrees remain in places. For example, the temperature gradient in the model from 1 to 0.1 mbar is not steep enough.

To fit more precisely the CIRS-derived temperatures from 4 to 0.2 mbar (Fig. 14), we used a slightly different vertical variation of the velocity profile with a maximum, expressed in pressure units, at 0.4-1 mbar (185-225 km), decreasing above and, more slowly, below this pressure range (thin and dashed lines in Fig. 11). This would imply (weak) convergence of the vertical flow below this region, and divergence above, a feature generally consistent with the upwelling branch of a Hadley cell (e.g. Lora et al. 2015). The two velocity profiles, derived from fitting of the $6^{\circ} \mathrm{N}$ profile retrieved by Vinatier et al. (2015) and of the CIRS temperature maps at selected levels (Fig. 15), differ by at most $40 \%$ in the $4-0.2$ mbar pressure range (at 1 mbar, $0.040 \mathrm{~cm} \mathrm{~s}^{-1}$ vs. $0.055 \mathrm{~cm} \mathrm{~s}^{-1}$ ). This difference lies in the uncertainty area indicated in Fig. 9 and results from small systematic differences (typically 1-3 K) in the retrievals from Achterberg et al. (in preparation) and Vinatier et al. (2015). These differences partly result from the use of different sets of spectra (respectively nadir spectra at $2.8-\mathrm{cm}^{-1}$ resolution and a combination of nadir and limb spectra at $0.5-\mathrm{cm}^{-1}$ resolution) and the different vertical resolution of the retrievals.

\subsection{Seasonal variations of temperatures from 0.2 to 4 mbar}


743 At equatorial latitudes, we found that the modulation of solar heating due to Saturn's orbital

744 eccentricity is large enough to explain the $\sim 4 \mathrm{~K}$ drop between pre-equinox (2006-2008) and

745 mid-2016 temperature at 1 mbar. The Sun-Saturn distance increased from 9.2 to 10.0 AU

746 between January 2007 and January 2017, causing a decrease in insolation of $17 \%$. This

747 induces radiatively a $\sim 7 \mathrm{~K}$ drop in the 1-mbar temperature (Fig. 10). To reduce this drop to

748 the observed variation, a modulation of the adiabatic cooling is needed, with less cooling after

749 equinox than before. Assuming for $\operatorname{simplicity}$ a $\sin \left(L_{s}\right)$ variation for this modulation, the

750 upward velocity at 1 mbar in our best fit model varies between 0.035 and $0.075 \mathrm{~cm} \mathrm{~s}^{-1}$ over a

751 Titan year.

752

753 At $30^{\circ} \mathrm{S}$ and $30^{\circ} \mathrm{N}$, we also find that our radiative model with no modulation of the adiabatic

754 cooling considerably overestimates the observed seasonal variation at all pressure levels

755 between 0.2 and 4 mbar. Assuming that the vertical velocity at a given level is an affine

756 function of $\sin \left(L_{s}\right)$ (Eq. 8) and that its vertical variation is the same at all latitudes, the

757 observed variations of temperature are relatively well reproduced with the parameters listed in

758 Table 1 . An exception is the 4-mbar temperatures at the equator and $30^{\circ} \mathrm{N}$, which show a

759 steady decrease with time since the beginning of the Cassini mission. Such a variation cannot

760 be reproduced with our simple radiative-dynamical model and would require a functional

761 form for the time variation of the vertical velocity more complex than assumed in Eq. 8 .

763 Our model shows that the time variation of the adiabatic cooling acts to counterbalance the

764 seasonal variations of solar heating, with enhanced upwelling in summer. The modulation is

765 stronger at $30^{\circ} \mathrm{S}$ than at $30^{\circ} \mathrm{N}$, likely because the seasonal variations of solar heating are more

766 pronounced in the southern hemisphere than in the northern one due to the eccentricity of

767 Saturn's orbit, perihelion occurring less than a year after southern summer solstice. At the 
equator, the relatively weak modulation of the adiabatic cooling $(\sim 35 \%)$ acts to mitigate the variation of solar heating due to the eccentricity, with enhanced adiabatic cooling in northern winter / southern summer when the Sun-Saturn distance is smaller. At $30^{\circ} \mathrm{N}$ and more significantly at $30^{\circ} \mathrm{S}$, our model predicts downwelling, and thus compressional heating, around winter solstice. At $30^{\circ} \mathrm{S}$, the predicted maximum downward velocity at $0.5-1 \mathrm{mbar}$ is $0.05 \mathrm{~cm} \mathrm{~s}^{-1}$. This value is actually close to the downward velocity of $0.06 \mathrm{~cm} \mathrm{~s}^{-1}$ at $1 \mathrm{mbar}$ required to balance the radiative cooling of $0.06 \mathrm{~K} \mathrm{day}^{-1}$ derived by Teanby et al. (2017) near $80^{\circ} \mathrm{S}$ in January 2016 . This would imply that, around winter solstice, subsidence is not limited to winter polar latitudes but extends equatorward to at least $\sim 30^{\circ}$. Conversely, around equinox, for $L_{\mathrm{s}}$ between approximately $320^{\circ}$ and $30^{\circ}$, upwelling occurs over the whole latitude range $30^{\circ} \mathrm{S}-30^{\circ} \mathrm{N}$.

\section{Conclusions}

We have developed a one-dimensional seasonal radiative-dynamical model of Titan's atmosphere to investigate the temporal variations of Titan's stratospheric temperatures observed from 2004 to 2016 by Cassini/CIRS. This model calculates the radiative forcing using gas and haze vertical opacity profiles constrained by Cassini/CIRS and Huygens/DISR measurements. Adiabatic heating and cooling can occur by introducing a vertical velocity $(w)$ in the energy equation. Applying this model to low latitudes, we conclude that:

- The heating and cooling rate profiles we obtained at $20^{\circ} \mathrm{S}$ are in good agreement with those calculated by Tomasko et al. (2008b). While the heating and cooling rates both decrease by a factor of $\sim 20$ from 0.1 to 5 mbar, the heating rate constantly exceeds the cooling rate by $20-35 \%$ over this range. We find that the net radiative heating minus cooling rate steadily increase with height from the troposphere up to $250 \mathrm{~km}$, in contrast with Tomasko et al. (2008b) who suggested a maximum near $120 \mathrm{~km}$. 
- The radiative time constant $\left(\tau_{R}\right)$ associated with the damping of a small temperature perturbation, one-scale height broad, is typically one order of magnitude shorter than that simply estimated by dividing the temperature by the cooling rate (Strobel et al. 2009). Our radiative time constant is also significantly shorter than that estimated by Achterberg et al. (2011) using the cooling-to-space approximation and neglecting aerosol emission. We find that $\tau_{R}$ exceeds $10^{9} \mathrm{~s}$ (i.e. a Titan year) only in the first half scale height of Titan's atmosphere, contrary to previous estimations. Above the 5mbar level, $\tau_{R}$ is so short, less than 0.02 Titan year, that the solar heating rate and radiative plus dynamical cooling rate are essentially balanced at any time.

- At $6^{\circ} \mathrm{N}$ around equinox, where seasonal variations should be at minimum, we find that, 803 as at $20^{\circ} \mathrm{S}$, the heating rate exceeds the cooling rate at all altitudes and the radiative solution profile is thus warmer than the observed one. Adding adiabatic cooling with an upward velocity of $\sim-1$ to $-1.5 \mathrm{~Pa} /$ Titan day (in pressure units) up to $375 \mathrm{~km}(0.02$ mbar), brings the model profile in general agreement with the observed one. The corresponding velocity $w$ varies thus approximately as the inverse of the atmospheric density in this range, with $w \sim 0.03-0.05 \mathrm{~cm} \mathrm{~s}^{-1}$ at $1 \mathrm{mbar}(185 \mathrm{~km})$ and reaching its maximum, of the order of $1 \mathrm{~cm} \mathrm{~s}^{-1}$, around $0.01 \mathrm{mbar}(415 \mathrm{~km})$. Our model is however affected by uncertainties in the haze density profile above $\sim 225 \mathrm{~km}$.

- Variations of solar heating due to Saturn's orbit eccentricity are more than sufficient to cause the decrease of equatorial temperatures observed from pre-equinox to 2016 . A weak seasonal modulation of $w(\sim 35 \%)$ is needed to bring the radiative model variation $(\sim 7 \mathrm{~K}$ at $1 \mathrm{mbar})$ near the observed one ( $\sim \mathrm{K}$ at $1 \mathrm{mbar})$. This modulation reduces the adiabatic cooling during northern summer, i.e. when the Sun-Saturn distance is larger, and enhances it during southern summer. 
- The seasonal variations predicted by the radiative model with constant-with-time adiabatic cooling are also much larger than observed at $30^{\circ} \mathrm{S}$ and $30^{\circ} \mathrm{N}$. A simple modulation of the vertical velocity profile as an affine function of $\sin \left(L_{s}\right)$ is capable of correctly reproducing the observed variations. While the required year-averaged velocity is upward (providing adiabatic cooling) at $0,30^{\circ} \mathrm{S}$ and $30^{\circ} \mathrm{N}$, subsidence is predicted to occur around winter solstice at $30^{\circ} \mathrm{S}$ and marginally at $30^{\circ} \mathrm{N}$. At 1 mbar, $w$ varies in the range $0.035-0.075 \mathrm{~cm} \mathrm{~s}^{-1}$ at the equator, $-0.05-0.16 \mathrm{~cm} \mathrm{~s}^{-1}$ at $30^{\circ} \mathrm{S}$ and

These results may serve as a guide to general Circulation Models of Titan, which generally incorporate a less precise treatment of the radiative forcing. Although our radiative cooling and heating profiles are constrained by Cassini/Huygens observations, they still suffer from uncertainties in the haze opacity profile in the upper stratosphere and mesosphere. In particular, we have not considered variations with season of the haze profile at low latitudes,

831 such as the evolution of the detached haze layer. We have also neglected the small seasonal 832 variations of gaseous composition that may occur at $30^{\circ} \mathrm{N}$ or $\mathrm{S}$. Nevertheless, the information given here should provide the basic radiative forcing at low latitudes for dynamical models.

We plan to extend this analysis to high latitudes where strong seasonal variations of

836 composition and temperature are observed (e.g. Teanby et al. 2012, Vinatier et al. 2015,

837 Coustenis et al. 2016, Teanby et al. 2017). The strong enhancement of photochemical species 838 that takes place in the stratosphere during winter strongly affects the cooling rate profile and 839 thus potentially the temperature profile. They clearly must be taken into account to 840 characterize the adiabatic heating needed to balance the net radiative cooling, especially 841 during the polar night. 
Acknowledgments

847 BB and SV acknowledge support from the Centre National d'Études Spatiales (CNES) and

848 the Programme National de Planétologie (INSU/CNRS). RKA was supported by the NASA 849 Cassini Project. We thank the two referees whose thorough reviews helped to improve the 850 paper. 


\section{References}

852

853 Achterberg, R. K., Conrath, B. J., Gierasch, P. J., Flasar, F. M., Nixon, C. A., 2008a. Titan’s 854 middle-atmospheric temperatures and dynamics observed by the Cassini Composite Infrared 855 Spectrometer. Icarus 194, 263-277. doi: 10.1016/j.icarus.2007.09.029.

856

857 Achterberg, R. K., Conrath, B. J., Gierasch, P. J., Flasar, F. M., Nixon, C. A., 2008b.

858 Observation of a tilt of Titan's middle-atmospheric superrotation. Icarus 197, 549-555. doi:

859 10.1016/j.icarus.2008.05.014.

860

861 Achterberg, R. K., Gierasch, P. J., Conrath, B. J., Flasar, F. M., Nixon, C. A., 2011. Temporal 862 variations of Titan's middle-atmospheric temperatures from 2004 to 2009 observed by

863 Cassini/CIRS. Icarus 211, 686-698. doi: 10.1016/j.icarus.2010.08.009.

864

865 Anderson, C. M., Samuelson, R. E., 2011. Titan's aerosol and stratospheric ice opacities

866 between 18 and $500 \mu \mathrm{m}$ : Vertical and spectral characteristics from Cassini CIRS. Icarus 212, 867 762-768. doi: 10.1016/j.icarus.2011.01.024.

868

869 Bézard, B., Coustenis, A., McKay, C. P., 1995. Titan's stratospheric temperature asymmetry: 870 A radiative origin? Icarus 113, 267-276. doi: 10.1006/icar.1995.1023.

871

872 Bézard, B., Nixon, C. A., Kleiner, I., Jennings, D. E., 2007. Detection of ${ }^{13} \mathrm{CH}_{3} \mathrm{D}$ on Titan.

873 Icarus 191, 397-400. doi: 10.1016/j.icarus.2007.06.004.

874 
875 Coustenis, A., et al., 2013. Evolution of the stratospheric temperature and chemical

876 composition over one Titanian year. Astrophys. J. 779, id. 177, 9 pp. doi: 10.1088/0004$877 \quad 637 \mathrm{X} / 779 / 2 / 177$

878

879 Coustenis, A., et al., 2016. Titan's temporal evolution in stratospheric trace gases near the 880 poles. Icarus 270, 409-420. doi: 10.1016/j.icarus.2015.08.027.

881

882 De Kok, R., et al., 2007. Oxygen compounds in Titan's stratosphere as observed by Cassini

883 CIRS. Icarus 186, 354-363. doi: 10.1016/j.icarus.2006.09.016.

884

885 Doose, L. R., Karkoschka, E., Tomasko, M. G., Anderson, C. M., 2016. Vertical structure and 886 optical properties of Titan's aerosols from radiance measurements made inside and outside 887 the atmosphere. Icarus 270, 355-375. doi: 10.1016/j.icarus.2015.09.039.

888

889 Flasar, F. M., Conrath, B. J. 1990. Titan's stratospheric temperatures: A case for dynamical 890 inertia? Icarus 85, 346-354. doi: 10.1016/0019-1035(90)90122-P.

891

892 Flasar, F. M., et al., 2004. Exploring the Saturn system in the thermal infrared: The

893 Composite Infrared Spectrometer. Space Sci. Rev. 115, 169-297. doi: 10.1007/s11214-004$894 \quad 1454-9$.

895

896 Flasar, F. M., Achterberg, R. K., Schinder, P. J., 2014. Thermal structure of Titan's

897 troposphere and middle atmosphere. In: Müller-Wodarg, I., Griffith, C. A., Lellouch, E.,

898 Cravens, T. E. (Eds.), Titan - Interior, Surface, Atmosphere and Space Environment.

899 Cambridge University Press, New York NY, pp. 102-121 (Chapter 3). 
901 Fulchignoni, M., et al., 2005. In situ measurements of the physical characteristics of Titan's environment. Nature 438, 785-791. doi: 10.1038/nature04314.

903

904 Hirtzig, M., et al., 2013. Titan's surface and atmosphere from Cassini/VIMS data with 905 updated methane opacity. Icarus 226, 470-486. doi: 10.1016/j.icarus.2013.05.033.

906 Corrigendum: Icarus 226, 1182. doi: 10.1016/j.icarus.2013.07.015.

907

908 Irwin, P. G. J., Calcutt, S. B., Taylor, F. W., Weir, A. L., 1996. Calculated $k$ distribution 909 coefficients for hydrogen- and self-broadened methane in the range $2000-9500 \mathrm{~cm}^{-1}$ from 910 exponential sum fitting to band-modelled spectra. J. Geophys. Res. 101, E11, 26137-26154. 911 doi: 10.1029/96JE02707.

912

913 Jacquemart, D., et al., 2008. New laboratory measurements of $\mathrm{CH}_{4}$ in Titan's conditions and a 914 reanalysis of the DISR near-surface spectra at the Huygens landing site. Planet. Space Sci. 56, 915 613-623. doi: 10.1016/j.pss.2007.10.008.

916

917 Jacquinet-Husson, N., et al., 2011. The 2009 edition of the GEISA spectroscopic database. J.

918 Quant. Spectrosc. Radiat. Trans. 112, 2395-2445. doi: 10.1016/j.jqsrt.2011.06.004. 919

920 Karkoschka, E., Lorenz, R. D., 1997. Latitudinal variation of aerosol sizes inferred from 921 Titan’s shadow. Icarus 125, 369-379. doi: 10.1006/icar.1996.5621. 
923 Karkoschka, E., Tomasko, M. G., 2010. Methane absorption coefficients for the jovian

924 planets from laboratory, Huygens, and HST data. Icarus 205, 674-694. doi:

925 10.1016/j.icarus.2009.07.044.

926

927 Karkoschka, E., Schröder, S. E., Tomasko, M. G., Keller, H. U., 2012. The reflectivity

928 spectrum and opposition effect of Titan's surface observed by Huygens' DISR spectrometers.

929 Planet. Space Sci. 60, 342-355. doi: 10.1016/j.pss.2011.10.014.

930

931 Landau, L., Lifchitz, E., 1969. Physique théorique. Tome I. Mécanique. $3^{\text {rd }}$ edition (revised).

932 Ed. MIR, Moscow.

933

934 Lebonnois, S., Burgalat, J., Rannou, P., Charnay, B., 2012. Titan global climate model: A

935 new 3-dimensional version of the IPSL Titan GCM. Icarus 218, 707-722. doi:

936 10.1016/j.icarus.2011.11.032.

937

938 Lebonnois, S., Flasar, F. M., Tokano, T., Newman, C. E., 2014. The general circulation of

939 Titan's lower and middle atmosphere. In: Müller-Wodarg, I., Griffith, C. A., Lellouch, E.,

940 Cravens, T. E. (Eds.), Titan - Interior, Surface, Atmosphere and Space Environment.

941 Cambridge University Press, New York NY, pp. 122-157 (Chapter 4).

942

943 Lellouch, E., et al., 2014. The distribution of methane in Titan's stratosphere from

944 Cassini/CIRS observations. Icarus 231, 323-337. doi: 10.1016/j.icarus.2013.12.016. 
Lora, J. M., Lunine, J. I., Russell, J. L. 2015. GCM simulations of Titan's middle and lower

947 atmosphere and comparison to observations. Icarus 250, 516-528. doi:

$948 \quad 10.1016 /$ j.icarus.2014.12.030.

949

950 Newman, C. E., Lee, C., Lian, Y., Richardson, M. I., Toigo, A. D., 2011. Stratospheric

951 superrotation in the TitanWRF model. Icarus 213, 636-654. doi: 10.1016/j.icarus.2011.03.025.

952

953 Niemann, H. B., et al., 2010. Composition of Titan's lower atmosphere and simple surface 954 volatiles as measured by the Cassini-Huygens probe gas chromatograph mass spectrometer 955 experiment. J. Geophys. Res. 115, E12006. doi: 10.1029/2010JE003659.

956

957 Press, W. H., Teukolsky, S. A., Vetterling, W. T., Flannery, B. P., 1997. Numerical Recipes in 958 Fortran 77: The Art of Scientific Computing (Vol. 1 of Fortran Numerical Recipes).

959 Cambridge Univ. Press, New York NY (Chapter 15).

960

961 Rey, M., Nikitin, A. V., Bézard, B., Rannou, P., Coustenis, A., Tyuterev, V. G., 2017. New 962 accurate theoretical line lists of ${ }^{12} \mathrm{CH}_{4}$ and ${ }^{13} \mathrm{CH}_{4}$ in the $0-13400 \mathrm{~cm}^{-1}$ range: Application to 963 the modelling of methane absorption in Titan's atmosphere. Icarus, submitted.

964

965 Rothman, L. S., et al., 2013. The HITRAN2012 molecular spectroscopic database. J. Quant.

966 Spectrosc. Radiat. Trans. 130, 4-50. doi: 10.1016/j.jqsrt.2013.07.002.

967

968 Stamnes, K., Tsay, S-C., Wiscombe, W., Jayaweera, K., 1988. Numerically stable algorithm

969 for discrete-ordinate-method radiative transfer in multiple scattering and emitting layered

970 media. Appl. Opt. 27, 2502-2509. doi: 10.1364/AO.27.002502. 
972 Strobel, D. F., et al., 2009. Atmospheric structure and composition. In: Brown, R. H.,

973 Lebreton, J.-P., Waite, J. H. (Eds.), Titan from Cassini-Huygens. Springer, Dordrecht, pp.

$974 \quad 235-257$ (Chapter 10).

975

976 Teanby, N. A., et al., 2007. Vertical profiles of $\mathrm{HCN}, \mathrm{HC}_{3} \mathrm{~N}$, and $\mathrm{C}_{2} \mathrm{H}_{2}$ in Titan's atmosphere 977 derived from Cassini/CIRS data. Icarus 186, 364-384. doi: 10.1016/j.icarus.2006.09.024.

978

979 Teanby, N. A., et al., 2008. Global and temporal variations in hydrocarbons and nitriles in

980 Titan's stratosphere for northern winter observed by Cassini/CIRS. Icarus 193, 595-611. doi:

$981 \quad 10.1016 /$ j.icarus.2007.08.017.

982

983 Teanby, N. A., Irwin, P. G. J., de Kok, R., Nixon, C. A. 2009. Dynamical implications of 984 seasonal and spatial variations in Titan's stratospheric composition. Phil. Trans. Royal Soc. 985 367, 697-711. doi: 10.1098/rsta.2008.0164.

986

987 Teanby, N. A., et al., 2012. Active upper-atmosphere chemistry and dynamics from polar 988 circulation reversal on Titan. Nature 491, 732-735. doi: 10.1038/nature11611.

989

990 Teanby, N. A., et al., 2017. The formation and evolution of Titan's winter polar vortex. Nat.

991 Commun., in press.

992

993 Tomasko, M. G., Bézard, B., Doose, L., Engel, S., Karkoschka, E., 2008a. Measurements of

994 methane absorption by the descent imager/spectral radiometer (DISR) during its descent

995 through Titan's atmosphere. Planet. Space Sci. 56, 624-647. doi: 10.1016/j.pss.2007.10.009. 
997 Tomasko, M. G., Bézard, B., Doose, L., Engel, S., Karkoschka, E., Vinatier, S., 2008b. Heat 998 balance in Titan's atmosphere. Planet. Space Sci. 56, 648-659. doi: 10.1016/j.pss.2007.10.012. 999

1000 Tomasko, M. G., et al., 2008c. A model of Titan's aerosols based on measurements made 1001 inside the atmosphere. Planet. Space Sci. 56, 669-707. doi: 10.1016/j.pss.2007.11.019.

1002

1003

Vinatier, S., et al., 2007. Vertical abundance profiles of hydrocarbons in Titan's atmosphere at $100415^{\circ} \mathrm{S}$ and $80^{\circ} \mathrm{N}$ retrieved from Cassini/CIRS spectra. Icarus 188, 120-138. doi:

1005 10.1016/j.icarus.2006.10.031.

1006

1007 Vinatier, S., et al., 2010a. Analysis of Cassini/CIRS limb spectra of Titan acquired during the 1008 nominal mission. I. Hydrocarbons, nitriles and $\mathrm{CO}_{2}$ vertical mixing ratio profiles. Icarus 205, 1009 559-570. doi: 10.1016/j.icarus.2009.08.013.

1010

1011 Vinatier, S., et al., 2010b. Analysis of Cassini/CIRS limb spectra of Titan acquired during the 1012 nominal mission. II: Aerosol extinction profiles in the $600-1420 \mathrm{~cm}^{-1}$ spectral range. Icarus 1013 210, 852-866. doi: 10.1016/j.icarus.2010.06.024.

1014

1015 Vinatier, S., Rannou, P., Anderson, C. A., Bézard, B., de Kok, R., Samuelson, R. E., 2012.

1016 Optical constants of Titan's stratospheric aerosols in the $70-1500 \mathrm{~cm}^{-1}$ spectral range

1017 constrained by Cassini/CIRS observations. Icarus 219, 5-12. doi:

$1018 \quad$ 10.1016/j.icarus.2012.02.009.

1019 
1020 Vinatier, S., et al., 2015. Seasonal variations in Titan's middle atmosphere during the northern

1021 spring derived from Cassini/CIRS observations. Icarus 250, 95-115. doi:

$1022 \quad$ 10.1016/j.icarus.2014.11.019.

1023

1024 Vinatier, S., et al., 2016. Seasonal variations in Titan's stratosphere observed with

1025 Cassini/CIRS after the northern spring equinox. American Astronomical Society, DPS

1026 meeting \#48, id.509.09.

1027

1028 Vinatier, S., et al., 2017a. Study of Titan's fall southern stratospheric polar cloud composition

1029 with Cassini/CIRS: detection of benzene ice. Icarus, submitted.

1030

1031 Vinatier, S., et al., 2017b. Seasonal variations in Titan's stratosphere observed with

1032 Cassini/CIRS during northern spring. American Astronomical Society, DPS meeting \#49, 1033 id.304.03. 